\title{
Properly Edge-colored Theta Graphs in Edge-colored Complete Graphs
}

\author{
Ruonan $\mathrm{Li}^{1,2} \cdot$ Hajo Broersma ${ }^{2}$ Shenggui Zhang ${ }^{1}$ \\ Received: 21 March 2018 / Revised: 13 November 2018 / Published online: 28 November 2018 \\ (c) The Author(s) 2018
}

\begin{abstract}
With respect to specific cycle-related problems, edge-colored graphs can be considered as a generalization of directed graphs. We show that properly edge-colored theta graphs play a key role in characterizing the difference between edge-colored complete graphs and multipartite tournaments. We also establish sufficient conditions for an edgecolored complete graph to contain a small and a large properly edge-colored theta graph, respectively.
\end{abstract}

Keywords Edge-colored graph - Complete graph · Properly edge-colored cycle . Properly edge-colored theta graph · Multipartite tournament

Mathematics Subject Classification $05 \mathrm{C} 15 \cdot 05 \mathrm{C} 20 \cdot 05 \mathrm{C} 38$

\section{Introduction}

All graphs considered in this paper are finite, simple, and undirected unless specified explicitly as directed graphs. For terminology and notation not defined here, we refer the reader to [3].

Let $G$ be a graph with vertex set $V(G)$ and edge set $E(G)$. For a proper subset $S$ of $V(G)$, we use $G-S$ to denote the subgraph of $G$ induced by $V(G) \backslash S$. For an

Supported by CSC (No. 201506290097) and NSFC (No. 11671320).

Hajo Broersma

h.j.broersma@utwente.nl

Ruonan Li

rnli@nwpu.edu.cn

Shenggui Zhang

sgzhang@nwpu.edu.cn

1 Department of Applied Mathematics, Northwestern Polytechnical University, Xi' an 710072, People's Republic of China

2 Faculty of EEMCS, University of Twente, P.O. Box 217, 7500 AE Enschede, The Netherlands 
edge $u v \in E(G), G-u v$ is the graph with vertex set $V(G)$ and edge set $E(G) \backslash\{u v\}$. For a proper subgraph $H$ of $G$, we use $G-H$ to denote the graph $G-V(H)$. An edge-coloring of $G$ is a mapping col : $E(G) \mapsto \mathbb{N}$, where $\mathbb{N}$ is the set of natural numbers. For $a, b \in \mathbb{N}$ with $a \leq b$, we use $[a, b]$ to denote $\{i \in \mathbb{N} \mid a \leq i \leq b\}$.

A graph $G$ with an assigned edge-coloring is called an edge-colored graph (or throughout this paper simply a colored graph). We say that a colored graph $G$ is a properly colored graph (or PC graph for short) if each pair of adjacent edges (i.e., edges that have precisely one end vertex in common) in $G$ are assigned distinct colors. Let $G$ be a colored graph. For an edge $e \in E(G)$, we use $\operatorname{col}(e)$ to denote the color of $e$. For a subgraph $H$ of $G$, we denote by $\operatorname{col}(H)$ the set of colors that are assigned to the edges of $E(H)$. The cardinality of $\operatorname{col}(G)$ is called the color number of $G$. We say a color appears (at least $k$ times) at a vertex $v \in V(G)$ if it is assigned to at least one (at least $k$ ) of the edges incident with $v$. For a vertex $v \in V(G)$, we denote by $N_{G}^{c}(v)$ the set of colors that are assigned to the edges incident with $v$. We call $d_{G}^{c}(v)=\left|N_{G}^{c}(v)\right|$ the color degree of $v$, and we use $\delta^{c}(G)=\min \left\{d_{G}^{c}(v) \mid v \in V(G)\right\}$ to denote the minimum color degree of $G$. When there is no ambiguity, we often write $N^{c}(v)$ for $N_{G}^{c}(v)$ and $d^{c}(v)$ for $d_{G}^{c}(v)$.

The existence of PC cycles in different types of colored graphs has been studied extensively during the last decades. Early research on the existence of PC Hamilton cycles in fact dates back to the 1970s [2,4,5,21], but this topic has also attracted new interest more recently [1,19]. Similarly, the existence of PC triangles has been studied by different research groups during the same period as well [6,8,10-12]. These topics have been dealt with for general graphs $[6,9,11,12,16-18,23]$ but also for complete graphs $[1,2,4-8,10,14,15,19,21]$ and for complete bipartite graphs $[1,4,15]$. Moreover, the theory involved in the study of PC cycles in edge-colored graph is closely related to the theory of directed cycles in directed graphs. In several proofs of theorems related to PC cycles, the analogy with directed graphs has been applied, and these techniques have often been used in constructions of extremal examples or in dealing with extremal cases. In fact, in this sense edge-colored graphs can be regarded as a generalization of directed graphs. We recall the following constructions for supporting evidence of this view.

Construction 1.1 Let $D$ be a directed graph with vertex set $\left\{v_{1}, v_{2}, \ldots, v_{n}\right\}$. Color each $\operatorname{arc} e=v_{i} v_{j}$ with color $i$. Then, ignoring the orientation of the arcs, we obtain a colored graph $G$.

Construction 1.2 Let $D$ be a multipartite tournament with partite sets $V_{1}, V_{2}, \ldots, V_{t}$ and arc set $A(D)$. Construct a colored complete graph $G$ with $V(G)=V(D)$, as follows. Add edges $u v$ with color $i$ joining all vertex pairs $u, v \in V_{i}$, and add edges $u v$ with color $j$ if and only if $u v \in A(D)$ with $u \in V_{j}$ and $v \in V_{i}(i \neq j)$.

Construction 1.3 Let $G$ be a colored graph (not necessarily complete) admitting a mapping $f: V(G) \mapsto \operatorname{col}(G)$ such that $\operatorname{col}(u v)=f(u)$ or $\operatorname{col}(u v)=f(v)$ for each edge $u v \in E(G)$. Construct a directed graph $D$ with $V(D)=V(G)$ and $u v \in A(D)$ if and only if $\operatorname{col}(u v)=f(u)$ and $\operatorname{col}(u v) \neq f(v)$.

When Constructions 1.1, 1.2 and 1.3 apply, PC cycles in $G$ are in a one to one correspondence with directed cycles in $D$. In particular, using Construction 1.2, for vertices 
$u$ and $v$ belonging to the same partite set, the edge $u v$ is not contained in any PC cycles in $G$. These observations are implied by the following fact (the proof of which is obvious and omitted).

Fact 1.1 Let $G$ be a colored graph. If there exists a mapping $f: V(G) \mapsto \operatorname{col}(G)$ such that $\operatorname{col}(u v)=f(u)$ or $\operatorname{col}(u v)=f(v)$ for each edge uv $\in E(G)$, then the following statements hold:

(i) G contains no PC cycle passing through an edge $e=x y$ satisfying $f(x)=f(y)$;

(ii) For each PC cycle $C=v_{1} v_{2} \ldots v_{\ell} v_{1}(\ell \geq 3)$ in $G$, either $f\left(v_{i}\right)=\operatorname{col}\left(v_{i} v_{i+1}\right)$ for all $i \in[1, \ell]$ or $f\left(v_{i}\right)=\operatorname{col}\left(v_{i} v_{i-1}\right)$ for all $i \in[1, \ell]$ (where the indices are taken modulo $\ell$ ).

Based on Fact 1.1 and Construction 1.2, we introduce the following definition.

Definition 1.1 A colored complete graph $G$ is essentially a multipartite tournament if there exists a mapping $f: V(G) \mapsto \operatorname{col}(G)$ such that $\operatorname{col}(u v)=f(u)$ or $\operatorname{col}(u v)=f(v)$ for each edge $u v \in E(G)$.

In [14], it is revealed that if a colored complete graph $G$ contains no monochromatic edge-cut and there exists a vertex $v$ which is not contained in any PC cycles in $G$, then a substructure of $G$ is essentially a multipartite tournament. Based on the above observations on the intimate relationship between colored graphs and directed graphs, one may wonder what the actual difference is between these two classes of graphs. This was our main motivation to study the difference between colored complete graphs and multipartite tournaments. It turns out that PC theta graphs play a key role in characterizing this difference.

Definition 1.2 A theta graph $\Theta_{k, \ell, m}$ is a graph obtained by joining two vertices by three internally-disjoint paths of lengths $k, \ell$ and $m$. We use $\left\{v_{0} v_{1} \ldots v_{k}\right.$, $\left.u_{0} u_{1} \ldots u_{\ell}, w_{0} w_{1} \ldots w_{m}\right\}$ with $v_{0}=u_{0}=w_{0}$ and $v_{k}=u_{\ell}=w_{m}$ to denote a $\Theta_{k, \ell, m}$.

Note that in a theta graph we allow one of the paths to have length 1, i.e., to consist of one edge, but we do not allow multiple edges.

Observation 1.1 Let $G$ be a colored graph (not necessarily complete). If there exists a mapping $f: V(G) \mapsto \operatorname{col}(G)$ such that $\operatorname{col}(u v)=f(u)$ or $\operatorname{col}(u v)=f(v)$ for each edge uv $\in E(G)$, then $G$ contains no PC theta graph.

Proof Define a directed graph $D$ with $V(D)=V(G)$ and $u v \in A(D)$ if and only if $\operatorname{col}(u v)=f(u)$ and $\operatorname{col}(u v) \neq f(v)$. Clearly, $D$ is a multipartite digraph. Suppose now, to the contrary, that $G$ contains a subgraph $H$ which is a PC $\Theta_{k, \ell, m}$. Let $P=v_{0} v_{1} \ldots v_{k}, Q=u_{0} u_{1} \ldots u_{\ell}$ and $R=w_{0} w_{1} \ldots w_{m}$ be the three internallydisjoint PC paths in $H$ with $v_{0}=u_{0}=w_{0}$ and $v_{k}=u_{\ell}=w_{m}$. Then $P$ and $Q$ form a PC cycle $C$, which corresponds to a directed cycle in $D$ (by Fact 1.1). Without loss of generality, assume that $v_{0} v_{1} \in A(D)$. Then $u_{\ell} u_{\ell-1} \in A(D)$ and by the definition of $D$, we know that $f\left(v_{0}\right)=\operatorname{col}\left(v_{0} v_{1}\right)$ and $f\left(u_{\ell}\right)=\operatorname{col}\left(u_{\ell} u_{\ell-1}\right)$, i.e., $f\left(w_{0}\right)=\operatorname{col}\left(v_{0} v_{1}\right)$ and $f\left(w_{m}\right)=\operatorname{col}\left(u_{\ell} u_{\ell-1}\right)$. Since $\operatorname{col}\left(w_{0} w_{1}\right) \neq \operatorname{col}\left(v_{0} v_{1}\right)$, we have $\operatorname{col}\left(w_{0} w_{1}\right) \neq f\left(w_{0}\right)$. This means that $\operatorname{col}\left(w_{0} w_{1}\right)=f\left(w_{1}\right)$. Repeating this argumentation, we get that $\operatorname{col}\left(w_{m-1} w_{m}\right)=f\left(w_{m}\right)=\operatorname{col}\left(u_{\ell} u_{\ell-1}\right)$. This contradicts that $H$ is a PC theta graph. 
Observation 1.1 clearly implies the following: if a colored complete graph $G$ is essentially a multipartite tournament, then $G$ contains no PC theta graph. On the other hand, it is not difficult to observe that the reverse does not hold in general: not every colored complete graph without PC theta (sub)graphs is essentially a multipartite tournament (see Fig. 1a, b for an example). Before we go into more detail on the difference between colored complete graphs and multipartite tournaments and the role that PC theta graphs play in this, we first introduce another definition and some observations.

Definition 1.3 Let $G$ be a colored graph. If $\delta^{c}(G-S)<\delta^{c}(G)$ for each nonempty proper subset $S \subset V(G)$, then we say $G$ is color degree critical (or $C D$-critical for short).

Obviously, the only CD-critical colored complete graph of minimum color degree 1 is a colored $K_{2}$. We also obtain a clear structure for CD-critical colored complete graphs with minimum color degree 2 . This structure is based on the following observation on the existence of small PC cycles in colored complete graphs.

Observation 1.2 (Li et al. [14]) Let $G$ be a colored complete graph with $\delta^{c}(G) \geq 2$. Then G contains a PC cycle of length 3 or 4 .

Observation 1.3 Let $G$ be a CD-critical colored complete graph with $\delta^{c}(G)=2$. Then $G$ is either a PC triangle or one of the graphs in Fig. 1.

Proof Let $G$ be a CD-critical colored complete graph with $\delta^{c}(G)=2$. Then, by using Observation 1.2, we know that $G$ contains either a PC triangle or a PC cycle of length 4. Since a PC cycle has minimum color degree 2, using Definition 1.3, we conclude that $G$ is either a PC triangle or a colored $K_{4}$ containing a PC cycle of length 4 . If $G \cong K_{4}$ and $G$ contains a monochromatic edge-cut, then $G$ must be isomorphic to the graph in Fig. 1a or b. The remaining case is that $G \cong K_{4}$, and $G$ contains no monochromatic edge-cut and no PC triangle. It is easy to verify that $G$ must be isomorphic to the graph in Fig. 1c.

Our main result characterizes the difference between CD-critical colored complete graphs and essentially multipartite tournaments in terms of the (non)existence of PC theta graphs, in the following way.

Theorem 1.4 Let $G$ be a $C D$-critical colored complete graph. Then $G$ contains no PC theta graph if and only if $G$ is essentially a multipartite tournament, unless $\delta^{c}(G)=2$ and $G$ is a colored $K_{4}$ containing a monochromatic edge-cut.

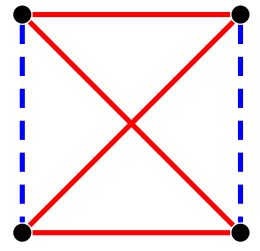

(a)

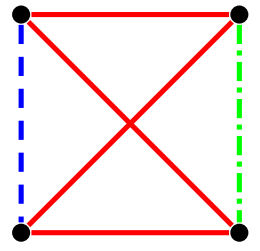

(b)

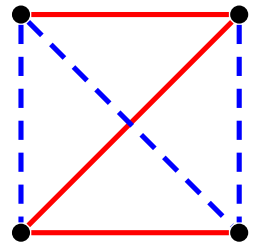

(c)

Fig. 1 CD-critical $K_{4}$ 's with minimum color degree 2. Here, different shadings indicate different colors 
In the exceptional case with $\delta^{c}(G)=2, G$ is either isomorphic to the graph in Fig. 1a or to the graph in Fig. 1b, as we have seen earlier. We postpone the rest of the proof of Theorem 1.4 to Sect. 3. The following infinite class of examples shows that, in Theorem 1.4, the restriction that $G$ is CD-critical cannot be omitted.

Example 1.1 Let $F$ be isomorphic to the graph in Fig. 1a. Given integers $t \geq 3$ and $n \geq 2 t-1$, let $T$ be a tournament satisfying $V(T)=\left\{v_{1}, v_{2}, \ldots, v_{n}\right\}, \delta^{-}(T)=t-1$ and $\delta^{+}(T) \geq 1$. Using Construction 1.1, we can construct a colored complete graph $H$ from $T$. Let $G$ be the colored complete graph obtained by joining $H$ and $F$ (adding all edges between vertices of $H$ and vertices of $F)$ such that $\operatorname{col}\left(u v_{i}\right)=i$ for all $u \in V(F)$ and $i \in[1, n]$.

In Example 1.1, since $H$ is an induced subgraph of $G$ and $\delta^{c}(H)=\delta^{c}(G)$, the graph $G$ is clearly not CD-critical. It is easy to verify that $G$ is not a colored $K_{4}$ and that $G$ contains no PC theta graph (by observing that $F$ and $H$, respectively, contain no PC theta graph, and every edge between $F$ and $H$ is not contained in any PC cycles). It is also easy to check that there is no mapping $f: V(G) \mapsto \operatorname{col}(G)$ such that $\operatorname{col}(u v)=f(u)$ or $\operatorname{col}(u v)=f(v)$ for each edge $u v \in E(G)$. So, $G$ is not essentially a multipartite tournament.

We conclude this introductory section with two other observations that motivated our interest in the existence of PC theta graphs. Let $x, y$ be the two vertices of degree 3 in a PC theta graph $\Theta_{k, \ell, m}$. Then there are three internally-disjoint PC paths between $x$ and $y$ with starting colors distinct and ending colors distinct. This can be regarded as "local PC connectivity", analogous to the concept of "local connectivity" in undirected graphs (without an edge-coloring). This "local PC connectivity" can help forming larger PC structures, in the following sense. Firstly, consider one PC theta graph $H$ in a colored complete graph $G$. Assume that $P, Q$ and $R$ are the three internallydisjoint PC paths in $H$. Then it is easy to verify that for each pair of distinct vertices $x, y \in V(G) \backslash V(H)$, one of $x P y, x Q y$ and $x R y$ is a PC path. Secondly, suppose we have vertex-disjoint PC theta graphs $H_{1}, H_{2}, \ldots, H_{k}$ in a colored complete graph $G$, and let $x_{i}, y_{i} \in V\left(H_{i}\right)$ satisfy $d_{H_{i}}\left(x_{i}\right)=d_{H_{i}}\left(y_{i}\right)=3$ for all $i \in[1, k]$. Then, it is again easy to verify that there exists a PC cycle in $G$ containing $\cup_{i=1}^{k}\left\{x_{i}, y_{i}\right\}$. Based on these observations, the existence of PC theta graphs (of small order) might have some implications for finding large PC cycles.

The rest of the paper is organized as follows. In the next section, some additional terminology and notation will be introduced, as well as some auxiliary lemmas that we need for our proof of Theorem 1.4. This proof is presented in Sect. 3.

In Sect. 4, we present and prove the following result, involving a sufficient color number condition for the existence of small PC theta graphs in colored complete graphs. Let $G$ be a colored $K_{n}$. If $|\operatorname{col}(G)| \geq n+1$, then $G$ contains a PC $\Theta_{1,2,2}$ or a PC $\Theta_{1,2,3}$. We also discuss the tightness of the condition.

In Sect. 5, the following color degree condition for the existence of large PC theta graphs is obtained. Let $G$ be a colored $K_{n}$. If $\delta^{c}(G) \geq \frac{n+1}{2}$, then one of the following statements holds:

(i) $d^{c}(u)=\frac{n+1}{2}$ for each vertex $u \in V(G)$ and $G$ contains a PC Hamilton cycle;

(ii) each maximal PC cycle $C$ in $G$ has a chord $u v$ such that $\left\{u v, u C^{+} v, u C^{-} v\right\}$ is a PC theta graph. 
This result is related to the following conjecture of Fujita and Magnant [7]: Let $G$ be a colored $K_{n}$. If $\delta^{c}(G) \geq \frac{n+1}{2}$, then each vertex of $G$ is contained in a PC cycle of length $\ell$ for all $\ell \in[3, n]$. Our result also indicates a possible approach to obtaining results on the existence of PC Hamilton cycles in colored complete graphs. As a consequence, we obtain the following result. Let $G$ be a colored $K_{n}$. If $\delta^{c}(G) \geq \frac{n}{2}+1$, then each vertex of $G$ is contained in a PC theta graph $\Theta_{1, k, m}$ such that $k+m \geq \delta^{c}(G)$.

We conclude the paper in Sect. 6 with some additional remarks and open questions.

\section{Preliminaries}

Let $G$ be a graph and $H$ a subgraph of $G$. We use $G[H]$ to denote the subgraph of $G$ induced by $V(H)$. Let $C$ be a cycle with a fixed orientation. For vertices $x, y \in V(C)$, $x C^{+} y$ denotes the segment on $C$ from $x$ to $y$ along the direction specified by the orientation of $C$, and $x C^{-} y$ the segment on $C$ along the reverse direction. For a vertex $v$ on $C$, denote by $v^{+}$and $v^{-}$the immediate successor and predecessor of $v$ on $C$, respectively. We set $v^{++}=\left(v^{+}\right)^{+}$and $v^{--}=\left(v^{-}\right)^{-}$. Similarly, for vertices $x$ and $y$ on a path $P$, the segment on $P$ between $x$ and $y$ is denoted by $x P y$. A graph obtained from two disjoint cycles by joining them by one connecting path, by one edge, or by identifying two vertices is called a generalized bowtie (or g-bowtie for short). See Fig. 2 for the three possible structures of a g-bowtie that we distinguish.

Let $G$ be a colored graph.

For a color $i \in \operatorname{col}(G)$, we denote by $G^{i}$ the spanning subgraph of $G$ with edge set $\{e \mid \operatorname{col}(e)=i\}$. For disjoint subsets $S$ and $T$ of $V(G)$, we let $E_{G}(S, T)$ be the set of edges between $S$ and $T$, and let $\operatorname{col}_{G}(S, T)$ be the set of colors that are appearing on $E_{G}(S, T)$ in $G$. If $S=\{v\}$, we often write $E_{G}(v, T)$ and $\operatorname{col}_{G}(v, T)$, respectively, instead of $E_{G}(\{v\}, T)$ and $\operatorname{col}_{G}(\{v\}, T)$. For two vertex-disjoint subgraphs $F$ and $H$ of $G$, we use $\operatorname{col}_{G}(F, H)$ to denote $\operatorname{col}_{G}(V(F), V(H))$. When there is no ambiguity, we often write $\operatorname{col}(S, T)$ for $\operatorname{col}_{G}(S, T)$ and $\operatorname{col}(F, H)$ for $\operatorname{col}_{G}(F, H)$. For each vertex $v \in V(G)$, we define

$$
\operatorname{Dom}_{G}(v)=\left\{u \in V(G) \mid d_{G-v}^{c}(u)=d_{G}^{c}(u)-1\right\}
$$

and

$$
\operatorname{Dom}_{G}^{*}(v)=\left\{u \in V(G) \mid d_{G-v}^{c}(u)=\delta^{c}(G)-1\right\},
$$

and we call these sets the dominating set and special dominating set of $v$ in $G$, respectively. Obviously, for each vertex $v \in V(G), \operatorname{Dom}_{G}^{*}(v) \subseteq \operatorname{Dom}_{G}(v)$.

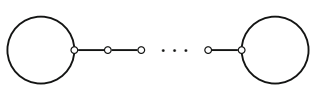

(a)

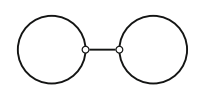

(b)

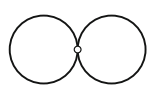

(c)

Fig. 2 Three different types of g-bowties 
We continue by presenting the following lemma that we need as a tool in the later proofs.

Note that we use $C_{1}$ and $C_{2}$ below (and also $C_{i}$ in the sequel) to denote arbitrary cycles, so $C_{i}$ does not indicate a cycle of length $i$ in this paper.

Lemma 2.1 Let $H$ be a PC g-bowtie in a colored complete graph G. Let $C_{1}, C_{2}$ and $P$ be the two cycles and the connecting path in $H$ with $V\left(C_{1}\right) \cap V(P)=\{x\}$ and $V\left(C_{2}\right) \cap V(P)=\{y\}$ (See Fig. 3). If $G[H]$ contains no PC theta graph and there exist an orientation of $C_{1}$ and a vertex $u$ on $C_{1}$ such that $u \in \operatorname{Dom}_{G[H]}\left(u^{-}\right)$, $u^{+} \in \operatorname{Dom}_{G[H]}(u)$ and $u, u^{+} \neq x$, then for each vertex $v \in V\left(C_{2}\right) \backslash\{y\}$ and each orientation of $\mathrm{C}_{2}$, the following three statements hold:

(i) $\operatorname{col}(v u)=\operatorname{col}\left(u u^{+}\right)$and $\operatorname{col}\left(v u^{+}\right)=\operatorname{col}\left(u^{+} u^{++}\right)$;

(ii) $\left\{\operatorname{col}(v u), \operatorname{col}\left(v u^{+}\right)\right\}=\left\{\operatorname{col}\left(v v^{+}\right), \operatorname{col}\left(v v^{-}\right)\right\}$;

(iii) $v \notin \operatorname{Dom}_{G[H]}\left(v^{+}\right) \cup \operatorname{Dom}_{G[H]}\left(v^{-}\right)$and $v \notin \operatorname{Dom}_{G}\left(v^{+}\right) \cup \operatorname{Dom}_{G}\left(v^{-}\right)$.

Proof Let $v$ be a vertex on $C_{2}$ distinct from $y$. Since $u \in \operatorname{Dom}_{G[H]}\left(u^{-}\right)$, we have $\operatorname{col}(v u) \neq \operatorname{col}\left(u^{-} u\right)$. Suppose that $\operatorname{col}(v u) \neq \operatorname{col}\left(u u^{+}\right)$. Since $\operatorname{col}\left(v v^{-}\right) \neq \operatorname{col}\left(v v^{+}\right)$, either $\left\{x C_{1}^{+} u, x C_{1}^{-} u, x P y C_{2}^{+} v\right\}$ or $\left\{x C_{1}^{+} u, x C_{1}^{-} u, x P y C_{2}^{-} v\right\}$ is a PC theta graph, a contradiction. So we have $\operatorname{col}(v u)=\operatorname{col}\left(u u^{+}\right)$. Similarly, we obtain $\operatorname{col}\left(v u^{+}\right)=\operatorname{col}\left(u^{+} u^{++}\right)$. If there exists a vertex $w \in\left\{u, u^{+}\right\}$such that $\operatorname{col}(w v) \notin\left\{\operatorname{col}\left(v v^{+}\right), \operatorname{col}\left(v v^{-}\right)\right\}$, then $\left\{v w C_{1}^{-} x P y, v C_{2}^{+} y, v C_{2}^{-} y\right\}$ is a PC theta graph, a contradiction. So, we conclude that $\left\{\operatorname{col}(v u), \operatorname{col}\left(v u^{+}\right)\right\}=\left\{\operatorname{col}\left(v v^{+}\right)\right.$, $\left.\operatorname{col}\left(v v^{-}\right)\right\}$. Thus both the colors $\operatorname{col}\left(v v^{+}\right)$and $\operatorname{col}\left(v v^{-}\right)$appear at least twice at $v$ in $G[H]$ (and also in $G$ ), i.e., $v \notin \operatorname{Dom}_{G[H]}\left(v^{+}\right) \cup \operatorname{Dom}_{G[H]}\left(v^{-}\right)$and $v \notin \operatorname{Dom}_{G}\left(v^{+}\right)$ $\cup \operatorname{Dom}_{G}\left(v^{-}\right)$.

\section{Colored Complete Graphs Without PC Theta Graphs}

In this section, we present the proof of Theorem 1.4. For convenience, we repeat the statement of the theorem.

Theorem 1.4 Let $G$ be a $C D$-critical colored complete graph. Then $G$ contains no PC theta graph if and only if $G$ is essentially a multipartite tournament, unless $\delta^{c}(G)=2$ and $G$ is a colored $K_{4}$ containing a monochromatic edge-cut.

We already noticed that Observation 1.1 implies the following: if a colored complete graph $G$ is essentially a multipartite tournament, then $G$ contains no PC theta graph. So, this establishes the "if" part of Theorem 1.4. For the "only if" part, we deliver

Fig. 3 The structure of $H$ in Lemma 2.1

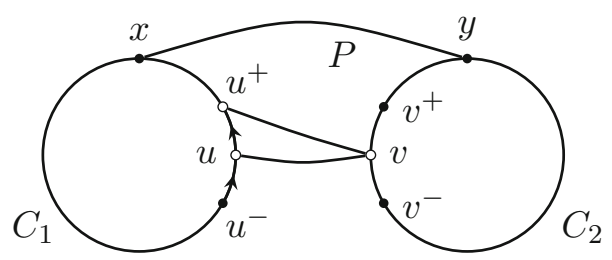


the proof in three steps. Lemma 3.1 below deals with the case that $\delta^{c}(G) \geq 3$ and $\left|\operatorname{col}\left(v^{*}, \operatorname{Dom}_{G}\left(v^{*}\right)\right)\right| \geq 2$ for some vertex $v^{*} \in V(G)$. Lemma 3.2 implies a structural property of $G$ when $\delta^{c}(G) \geq 3$ and $\left|\operatorname{col}\left(v, \operatorname{Dom}_{G}(v)\right)\right|=1$ for each vertex $v \in V(G)$. Based on this structural property, an auxiliary oriented graph is constructed, which helps to find a function $f$ in order to complete the proof of Theorem 1.4.

Since the two lemmas require rather long technical proofs, we first present the two lemmas without proofs, and then proceed to use them in order to prove Theorem 1.4. The remaining part of this section is then devoted to the proofs of the two lemmas.

Lemma 3.1 Let $G$ be a $C D$-critical colored complete graph with $\delta^{c}(G) \geq 3$. If $G$ contains no PC theta graph and $\left|\operatorname{col}\left(v^{*}, \operatorname{Dom}_{G}\left(v^{*}\right)\right)\right| \geq 2$ for some vertex $v^{*} \in V(G)$, then $G$ is essentially a multipartite tournament.

Lemma 3.2 Let $G$ be a $C D$-critical colored complete graph with $\delta^{c}(G) \geq 3$. If $G$ contains no PC theta graph and $\left|\operatorname{col}\left(v, \operatorname{Dom}_{G}(v)\right)\right|=1$ for each vertex $v \in V(G)$, then for each edge $x y \in E(G)$ satisfying $d_{G-x}^{c}(y)=\delta^{c}(G)-1$, we have $d_{G-y}^{c}(x) \geq \delta^{c}(G)$.

Next, we show how to prove Theorem 1.4 by applying Lemmas 3.1 and 3.2.

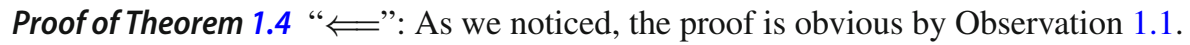

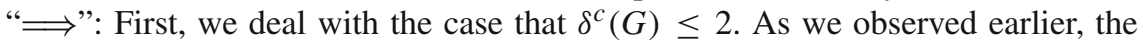
only CD-critical colored complete graph with $\delta^{c}(G)=1$ is a colored $K_{2}$, which is essentially a multipartite tournament. Let $G$ be a CD-critical colored complete graph with $\delta^{c}(G)=2$. Then by Observation $1.3, G$ must be a PC triangle or a colored $K_{4}$ as in Fig. 1. If $G$ is a PC triangle, then obviously, $G$ is essentially a multipartite tournament. If $G \cong K_{4}$ and $G$ contains no monochromatic edge-cut, then $G$ is the graph in Fig. 1c, which is essentially a multipartite tournament.

Now, assume that $\delta^{c}(G) \geq 3$. Since $G$ is CD-critical, for each vertex $v \in V(G)$, either $\left|\operatorname{col}\left(v, \operatorname{Dom}_{G}(v)\right)\right| \geq 2$ or $\left|\operatorname{col}\left(v, \operatorname{Dom}_{G}(v)\right)\right|=1$. If there exists a vertex $v \in V(G)$ with $\left|\operatorname{col}\left(v, \operatorname{Dom}_{G}(v)\right)\right| \geq 2$, then the proof is completed by directly applying Lemma 3.1. Next, we focus on the case that $\left|\operatorname{col}\left(v, \operatorname{Dom}_{G}(v)\right)\right|=1$ for each vertex $v \in V(G)$. Let $f(v)$ be the unique color in $\operatorname{col}\left(v, \operatorname{Dom}_{G}(v)\right)$. Since $G$ is CD-critical, for each vertex $v \in V(G)$, we actually have $\operatorname{Dom}_{G}^{*}(v) \neq \emptyset$.

Now define a directed graph $D$ with $V(D)=V(G)$ and $A(D)$ $=\left\{u v \mid v \in D_{o m}^{*}(u)\right\}$. Then $D$ is a directed graph with $\delta^{+}(D) \geq 1$ and for each arc $u v \in A(D)$, the color $\operatorname{col}(u v)=f(u)$ appears only once at $v$. Let $C_{1}, C_{2}, \ldots, C_{t}(t \geq 1)$ be a maximal collection of vertex-disjoint directed cycles in $D$ such that $D-\cup_{i=1}^{t} V\left(C_{i}\right)$ contains no directed cycles. By Lemma 3.2, we know that if $u v \in A(D)$, then $v u \notin A(D)$. Thus $\left|C_{i}\right| \geq 3$ for all $i \in[1, t]$. Let $R=V(G) \backslash\left(\cup_{i=1}^{t} V\left(C_{i}\right)\right)$ and let the direction of each cycle $C_{i}$ be the direction in $D$.

First we will show that $\operatorname{col}(u v)=f(u)$ or $\operatorname{col}(u v)=f(v)$ for each pair of distinct vertices $u, v \in \cup_{i=1}^{t} V\left(C_{i}\right)$. If $v$ is the successor of $u$ on a cycle $C \in\left\{C_{i} \mid i \in[1, t]\right\}$, then by the construction of $D$, we know that $v \in \operatorname{Dom}_{G}^{*}(u)$. Thus $\operatorname{col}(u v)=f(u)$. If $u$ and $v$ on a cycle $C \in\left\{C_{i} \mid i \in[1, t]\right\}$ are not consecutive vertices, then by considering the theta graph $\left\{u v, u C^{+} v, u C^{-} v\right\}$ (See Fig. 4a), we know that $\operatorname{col}(u v)=f(u)$ or $\operatorname{col}(u v)=f(v)$. Now let $u \in V\left(C_{i}\right)$ and $v \in V\left(C_{j}\right)$ for some $i, j \in[1, t]$ and 


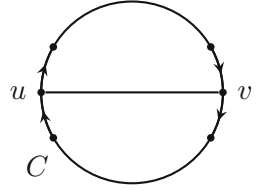

(a)

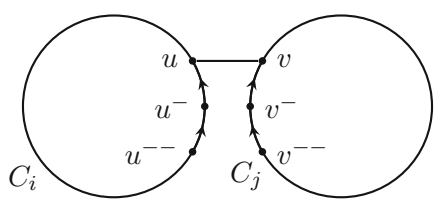

(b)

Fig. 4 Two cases when $u, v \in \cup_{i=1}^{t} V\left(C_{i}\right) \mathbf{a} u, v \in V\left(C_{i}\right), \mathbf{b} u \in C_{i}, v \in C_{j}$ and $i \neq j$

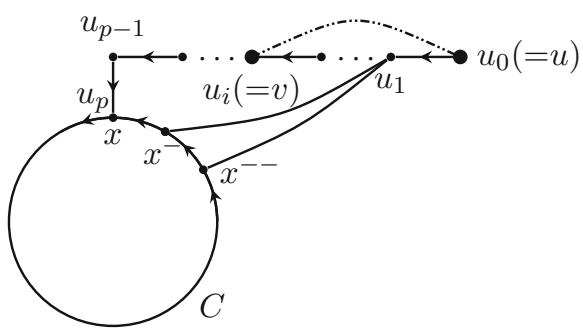

(a)

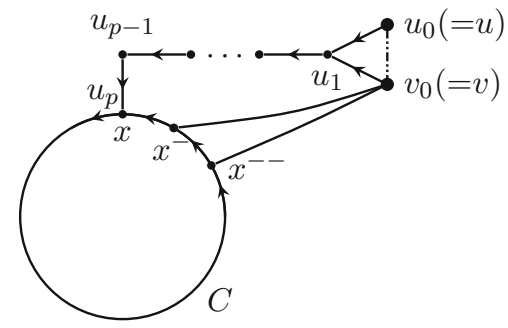

(b)

Fig. 5 Two cases when $V(P) \cap V(Q) \neq \emptyset \mathbf{a} v \in V(P), \mathbf{b} v \notin V(P), u \notin V(Q)$ and $u_{1}=v_{1}$

$i \neq j$. Suppose that $\operatorname{col}(u v) \neq f(u)$ and $\operatorname{col}(u v) \neq f(v)$ (See Fig. 4b). Then $C_{i}$, $C_{j}$ and the path $u v$ form a PC g-bowtie. By applying Lemma 2.1 to $v^{-}$, we obtain $v^{-} \notin \operatorname{Dom}_{G}\left(v^{--}\right)$, a contradiction. So $\operatorname{col}(u v)=f(u)$ or $\operatorname{col}(u v)=f(v)$ for each pair of distinct vertices $u, v \in \cup_{i=1}^{t} V\left(C_{i}\right)$.

If $R=\emptyset$, then the proof of Theorem 1.4 is already complete. Now assume that $R \neq \emptyset$. It remains to show that $\operatorname{col}(u v)=f(u)$ or $\operatorname{col}(u v)=f(v)$ for each pair of distinct vertices $u \in R$ and $v \in V(G)$. For each vertex $u \in R$, there must exist a directed path from $u$ to $\cup_{i=1}^{t} V\left(C_{i}\right)$ in $D$ (otherwise, since $\delta^{+}(D) \geq 1$, we can obtain a directed cycle in $D[R]$, a contradiction). Let $P=u_{0} u_{1} u_{2} \ldots u_{p}$ and $Q=v_{0} v_{1} v_{2} \ldots v_{q}$, respectively, be the directed paths in $D$ from $u$ to $\cup_{i=1}^{t} V\left(C_{i}\right)$ and from $v$ to $\cup_{i=1}^{t} V\left(C_{i}\right)$ with $u_{p}, v_{q} \in \cup_{i=1}^{t} V\left(C_{i}\right), u_{0}=u \in R$ and $v_{0}=v \in V(G)$. In particular, if $v \in \cup_{i=1}^{t} V\left(C_{i}\right)$, then $Q$ consists of the vertex $v$. We will show that $\operatorname{col}(u v)=f(u)$ or $\operatorname{col}(u v)=f(v)$ by considering the following three cases.

Case $1 V(P) \cap V(Q)=\emptyset$.

In this case, first suppose $u_{p}, v_{q} \in V(C)$ for some cycle $C \in\left\{C_{i} \mid i \in[1, t]\right\}$. Since $\left\{u_{p} C^{+} v_{q}, u_{p} C^{-} v_{q}, u_{p} P u_{0} v_{0} Q v_{q}\right\}$ is not a PC theta graph, we have $\operatorname{col}(u v)=f(u)$ or $\operatorname{col}(u v)=f(v)$. Next, suppose $u_{p} \in V\left(C_{i}\right)$ and $v_{q} \in V\left(C_{j}\right)$ for some $i, j \in[1, t]$ with $i \neq j$. Assume, to the contrary, that $\operatorname{col}(u v) \neq f(u)$ and $\operatorname{col}(u v) \neq f(v)$. Then $C_{i}, C_{j}$ and $u_{p} P u_{0} v_{0} Q v_{q}$ form a PC g-bowtie. Let $z$ be a vertex in $V\left(C_{j}\right) \backslash\left\{v_{q}\right\}$. Apply Lemma 2.1 to $z$. We obtain $z \notin \operatorname{Dom}_{G}\left(z^{-}\right)$, a contradiction.

Case $2 v \in V(P)$ or $u \in V(Q)$.

Assume that $v \in V(P)$. If $v=u_{1}$, then we have $\operatorname{col}(u v)=f(u)$. If $v=u_{i}$ for some $i \in[2, p]$ (See Fig. 5a), then $p \geq 2$. Assume that $u_{p} \in V(C)$ for some cycle 
$C \in\left\{C_{i} \mid i \in[1, t]\right\}$. Let $x=u_{p}$. Suppose, to the contrary, that $\operatorname{col}(u v) \neq f(u)$ and $\operatorname{col}(u v) \neq f(v)$. Then $u_{0} u_{1} \ldots u_{i} u_{0}$ is a PC cycle. Together with $C$ and the path $u_{i} u_{i+1} \ldots u_{p}$, we obtain a PC g-bowtie (it is possible that $u_{i}=u_{p}=x$ ). Apply Lemma 2.1 to $u_{1}$. We get $u_{1} \notin \operatorname{Dom}_{G}\left(u_{0}\right)$, a contradiction.

Case $3 v \notin V(P), u \notin V(Q)$ and $V(P) \cap V(Q) \neq \emptyset$.

In this case, assume that

there exist $i \in[1, p]$ and $j \in[1, q]$ such that $u_{i}=v_{j},\left\{u_{0}, u_{1}, \ldots, u_{i-1}\right\}$ $\cap V(Q)=\emptyset$ and $\left\{v_{0}, v_{1}, \ldots, v_{j-1}\right\} \cap V(P)=\emptyset$.

Let $C$ be the cycle in $\left\{C_{i} \mid i \in[1, t]\right\}$ such that $u_{p} \in V(C)$. Let $x=u_{p}$. Suppose, to the contrary, that $\operatorname{col}(u v) \neq f(u)$ and $\operatorname{col}(u v) \neq f(v)$. Then $v_{j} v_{j-1} \ldots v_{0} u_{0} u_{0} u_{1} \ldots u_{i-1} u_{i}$ is a PC cycle. Together with $C$ and the path $u_{i} u_{i+1} \ldots u_{p}$, we obtain a PC g-bowtie (it is possible that $v_{j}=u_{i}=u_{p}=x$ ). If $i \geq 2$, then apply Lemma 2.1 to $u_{1}$. We get $u_{1} \notin \operatorname{Dom}_{G}\left(u_{0}\right)$, a contradiction. If $j \geq 2$, then apply Lemma 2.1 to $v_{1}$. We get $v_{1} \notin \operatorname{Dom}_{G}\left(v_{0}\right)$, a contradiction. Now the only case left is that $i=j=1$ (See Fig. 5b). Apply Lemma 2.1 to $v_{0}$ and $u_{0}$, respectively. We get

$$
\left\{\operatorname{col}\left(x x^{-}\right), \operatorname{col}\left(x^{-} x^{--}\right)\right\}=\left\{\operatorname{col}\left(v_{0} u_{0}\right), \operatorname{col}\left(v_{0} u_{1}\right)\right\}
$$

and

$$
\left\{\operatorname{col}\left(x x^{-}\right), \operatorname{col}\left(x^{-} x^{--}\right)\right\}=\left\{\operatorname{col}\left(u_{0} v_{0}\right), \operatorname{col}\left(u_{0} u_{1}\right)\right\}
$$

Thus $\operatorname{col}\left(v_{0} u_{1}\right)=\operatorname{col}\left(u_{0} u_{1}\right)$. This contradicts that $u_{1} \in \operatorname{Dom}_{G}^{*}\left(u_{0}\right)$.

This completes the proof of Theorem 1.4.

In the remaining part of this section, we present the proofs of Lemmas 3.1 and 3.2.

Proof of Lemma 3.1 By contradiction. Suppose that $G$ is a counterexample to Lemma 3.1. Since $\left|\operatorname{col}\left(v^{*}, \operatorname{Dom}_{G}\left(v^{*}\right)\right)\right| \geq 2$, we can choose vertices $x, y \in \operatorname{Dom}_{G}\left(v^{*}\right)$ such that $\operatorname{col}\left(v^{*} x\right) \neq \operatorname{col}\left(v^{*} y\right)$. Let $\alpha=\operatorname{col}\left(v^{*} x\right)$ and $\beta=\operatorname{col}\left(v^{*} y\right)$. Then $\alpha$ and $\beta$ appear only once at $x$ and $y$, respectively. Let $c_{0}=\operatorname{col}(x y)$. Then $c_{0} \notin\{\alpha, \beta\}$. Considering the symmetry of $x$ and $y$, without loss of generality, assume that $d^{c}(x) \geq d^{c}(y)$. If $d^{c}(x)=d^{c}(y)$, then assume that $d_{G^{c}}(x) \leq d_{G^{c}}(y)$. Let $p=d^{c}(x)-2$. Since $\delta^{c}(G) \geq 3$, we have $p \geq 1$. Let $N^{c}(x)=\left\{\alpha, c_{0}, c_{1}, c_{2}, \ldots, c_{p}\right\}$. Define

$$
\begin{aligned}
S_{i} & =\left\{u \in V(G) \mid \operatorname{col}(x u)=c_{i}\right\} \text { for } i \in[1, p], \\
S_{0} & =\left\{u \in V(G) \backslash\{y\} \mid \operatorname{col}(x u)=c_{0}\right\}, \\
Z_{\alpha} & =\left\{z \in S_{0} \mid \operatorname{col}\left(v^{*} z\right)=\alpha\right\}, \\
Z_{\beta} & =\left\{z \in S_{0} \mid \operatorname{col}\left(v^{*} z\right)=\beta\right\}, \text { and } \\
Z_{0} & =\left\{z \in S_{0} \mid \operatorname{col}\left(v^{*} z\right)=c_{0}\right\} .
\end{aligned}
$$

Then $\left\{v^{*}, x, y\right\}, S_{0}, S_{1}, S_{2}, \ldots, S_{p}$ form a partition of $V(G)$, where it is not necessary but possible that $\beta \in\left\{c_{i} \mid i \in[1, p]\right\}$ and $S_{0}=\emptyset$. Since $S_{0}$ has a different property than $S_{i}(i \in[1, p])$, we often treat $S_{0}$ separately in the sequel. We will prove Lemma 
3.1 by analysing the colors between the parts. Our proof is based on a large number of claims, each of which is followed by a proof.

Claim $1 \operatorname{col}\left(y, S_{i}\right) \subseteq\left\{c_{0}, c_{i}\right\}$ and $\operatorname{col}\left(v^{*}, S_{i}\right) \subseteq\{\alpha, \beta\} \cup\left\{c_{i}\right\}$ for all $i \in[1, p]$.

Proof For $i \in[1, p]$ and a vertex $u \in S_{i}$, since $\left\{x y, x u y, x v^{*} y\right\}$ and $\left\{x v^{*}, x y v^{*}, x u v^{*}\right\}$ are not PC theta graphs, we have $\operatorname{col}(u y) \in\left\{c_{0}, c_{i}\right\}$ and $\operatorname{col}\left(u v^{*}\right) \in\{\alpha, \beta\} \cup\left\{c_{i}\right\}$.

Claim $2 \operatorname{col}\left(y, S_{0}\right) \subseteq\left\{c_{0}\right\}$, i.e., $\operatorname{col}\left(y, S_{0}\right)=\left\{c_{0}\right\}$ when $S_{0} \neq \emptyset$.

Proof Suppose to the contrary that there exists a vertex $z \in S_{0}$ such that $\operatorname{col}(y z) \neq c_{0}$. If there exists a vertex $v_{i} \in S_{i}$ for some $i \in[1, p]$ such that $\operatorname{col}\left(v_{i} y\right)=c_{0}$, then $\left\{x v^{*} y, x z y, x v_{i} y\right\}$ is a $\mathrm{PC} \Theta_{2,2,2}$, a contradiction. So by Claim 1, we have $\operatorname{col}\left(y, S_{i}\right)=\left\{c_{i}\right\}$ for all $i \in[1, p]$. Thus $\left\{c_{i} \mid i \in[0, p]\right\} \subseteq N^{c}(y)$. Note that $\beta$ appears only once at $y$. We have $\beta \notin\left\{c_{i} \mid i \in[0, p]\right\}$. Thus $d^{c}(y) \geq p+2=d^{c}(x)$ and

$$
\left\{u \in V(G) \mid \operatorname{col}(u y)=c_{0}\right\} \subseteq\left(S_{0}-z\right) \cup\{x\} .
$$

Recalling that $d^{c}(x) \geq d^{c}(y)$, we have $d^{c}(x)=d^{c}(y)$ and $d_{G^{c}}(y) \leq\left|S_{0}\right|<\left|S_{0}\right|$ $+1=d_{G^{c}}(x)$, a contradiction.

Claim 3 If there exists a vertex $u \in \cup_{i=1}^{p} S_{i}$ such that $\operatorname{col}(y u)=c_{0}$, then $\operatorname{col}\left(v^{*}, S_{0}\right)$ $\subseteq\left\{\alpha, \beta, c_{0}\right\}$ and $\operatorname{col}\left(S_{j}, S_{0}\right) \subseteq\left\{c_{j}, c_{0}\right\}$ for all $j \in[1, p]$.

Proof For a vertex $z \in S_{0}$, consider the theta graph $\left\{x v^{*}, x u y v^{*}, x z v^{*}\right\}$. We have $\operatorname{col}\left(z v^{*}\right) \in\left\{\alpha, \beta, c_{0}\right\}$. Thus $\operatorname{col}\left(v^{*}, S_{0}\right) \subseteq\left\{\alpha, \beta, c_{0}\right\}$. Suppose that there exist vertices $w \in S_{j}(j \in[1, p])$ and $z \in S_{0}$ such that $\operatorname{col}(w z) \notin\left\{c_{j}, c_{0}\right\}$. Then consider the color of $y w$. By Claim 1, we have $\operatorname{col}(y w) \in\left\{c_{j}, c_{0}\right\}$. If $\operatorname{col}(y w)=c_{0}$, then $\left\{x w, x z w, x v^{*} y w\right\}$ is a PC $\Theta_{1,2,3}$, a contradiction. If $\operatorname{col}(y w)=c_{j}$, then $w \neq u$ and $\left\{x u y, x v^{*} y, x z w y\right\}$ is a $\mathrm{PC} \Theta_{2,2,3}$, a contradiction. So, we have $\operatorname{col}\left(S_{j}, S_{0}\right) \subseteq\left\{c_{j}, c_{0}\right\}$ for all $j \in[1, p]$.

Claim $4 \operatorname{col}\left(S_{i}, S_{j}\right) \subseteq\left\{c_{i}, c_{j}\right\}$ for all $i, j \in[1, p]$ with $i<j$.

Proof By contradiction. Without loss of generality, suppose that there are vertices $v_{1} \in S_{1}$ and $v_{2} \in S_{2}$ such that $\operatorname{col}\left(v_{1} v_{2}\right)=a \notin\left\{c_{1}, c_{2}\right\}$. Since $\left\{x y, x v^{*} y, x v_{2} v_{1} y\right\}$ is not a PC theta graph and the color $\beta$ appears only once at $y$, we have $\operatorname{col}\left(y v_{1}\right) \neq c_{1}$. By Claim 1, $\operatorname{col}\left(y v_{1}\right)=c_{0}$. Similarly, we can obtain $\operatorname{col}\left(y v_{2}\right)=c_{0}$. Consider the theta graph $\left\{x v_{1}, x v_{2} v_{1}, x v^{*} y v_{1}\right\}$. We have $a=c_{0}$. Note that either $\beta \neq c_{1}$ or $\beta \neq c_{2}$. Without loss of generality, assume that $\beta \neq c_{1}$. Consider the color of $v_{1} v^{*}$. By Claim 1 , we know that $\operatorname{col}\left(v_{1} v^{*}\right) \in\left\{\alpha, \beta, c_{1}\right\}$. If $\operatorname{col}\left(v_{1} v^{*}\right)=\alpha$, then $\left\{x v_{1}, x v_{2} v_{1}, x y v^{*} v_{1}\right\}$ is a PC $\Theta_{1,2,3}$. If $\operatorname{col}\left(v_{1} v^{*}\right)=\beta$, then $\left\{x v_{1}, x v_{2} v_{1}, x v^{*} v_{1}\right\}$ is a PC $\Theta_{1,2,2}$. So we have $\operatorname{col}\left(v_{1} v^{*}\right)=c_{1}$. However, this implies that $\left\{x v^{*}, x y v^{*}, x v_{2} v_{1} v^{*}\right\}$ is a PC $\Theta_{1,2,3}$, a contradiction.

Claim $5 \operatorname{col}\left(G\left[S_{i}\right]\right)=\left\{c_{i}\right\}$ for all $i \in[1, p]$ and $\left|S_{i}\right| \geq 2$. 
Proof Suppose the contrary. Then the set $T=\left\{u_{i} w_{i} \in E\left(G\left[S_{i}\right]\right) \mid \operatorname{col}\left(u_{i} w_{i}\right)\right.$ $\left.\neq c_{i}, 1 \leq i \leq p\right\}$ is non-empty. Let $G^{\prime}=G-V(T)$. We will obtain a contradiction by proving that $\delta^{c}\left(G^{\prime}\right) \geq \delta^{c}(G)$ (this contradicts that $G$ is CD-critical). We do this by first proving two subclaims.

Subclaim $5.1 \operatorname{col}(y, V(T))=\left\{c_{0}\right\}, \operatorname{col}\left(v^{*}, V(T)\right) \subseteq\{\alpha, \beta\}$ and $\operatorname{col}\left(S_{i} \backslash V(T)\right.$, $V(T)) \subseteq\left\{c_{i}\right\}$ for all $i \in[1, p]$.

Proof Let $u$ be a vertex in $V(T)$ and let $u w$ be an edge in $T$. Without loss of generality, assume that $u, w \in S_{1}$. Consider the theta graph $\left\{x v^{*}, x y v^{*}, x w u v^{*}\right\}$. We know that either $\operatorname{col}\left(v^{*} u\right) \neq c_{1}$ or $\operatorname{col}\left(v^{*} u\right)=c_{1}=\beta$. Claim 1 tells that $\operatorname{col}\left(v^{*} u\right) \in\left\{\alpha, \beta, c_{1}\right\}$. Thus we will get $\operatorname{col}\left(v^{*} u\right) \in\{\alpha, \beta\}$ in both cases. Considering the theta graph $\left\{x y, x v^{*} y, x w u y\right\}$, we have $\operatorname{col}(y u) \neq c_{1}$. By Claim 1, we get $\operatorname{col}(y u)=c_{0}$. Thus $\operatorname{col}(y, V(T))=\left\{c_{0}\right\}$ and $\operatorname{col}\left(v^{*}, V(T)\right) \subseteq\{\alpha, \beta\}$. Consider a vertex $v_{i} \in S_{i} \backslash V(T)$ for some $i \in[1, p]$. If $i=1$, then by the definition of $T$ and the fact that $v_{i} \notin V(T)$, we have $\operatorname{col}\left(v_{i} w\right)=c_{i}$. Now consider the case that $i \neq 1$. Suppose that $\operatorname{col}\left(v_{i} u\right) \neq c_{i}$. By Claim 4 , we have $\operatorname{col}\left(v_{i} u\right)=c_{1}$. Consider the color of $y v_{i}$. If $\operatorname{col}\left(y v_{i}\right)=c_{0}$, then $\left\{x v_{i}, x v^{*} y v_{i}, x w u v_{i}\right\}$ is a PC $\Theta_{1,3,3}$, a contradiction. Otherwise, by Claim $1, \operatorname{col}\left(y v_{i}\right)=c_{i}$. This implies that $\left\{x y, x v^{*} y, x w u v_{i} y\right\}$ is a PC $\Theta_{1,2,4}$, a contradiction. So we have $\operatorname{col}\left(S_{i} \backslash V(T), V(T)\right) \subseteq\left\{c_{i}\right\}$ for all $i \in[1, p]$.

Since $T \neq \emptyset$ and $\operatorname{col}(y, V(T))=\left\{c_{0}\right\}$, by Claim 3, $\operatorname{col}\left(v^{*}, S_{0}\right) \subseteq\left\{\alpha, \beta, c_{0}\right\}$. Thus $S_{0}=Z_{\alpha} \cup Z_{\beta} \cup Z_{0}$.

Subclaim $5.2 \operatorname{col}\left(Z_{0}, V(T)\right) \subseteq\left\{c_{0}\right\}$ and $\operatorname{col}\left(Z_{\beta}, V(T)\right) \subseteq\left\{c_{0}, \beta\right\}$.

Proof Let $u$ be a vertex in $V(T)$ and let $u w$ be an edge in $T$. Assume that $u, w \in S_{i}$ for some $i \in[1, p]$. Suppose that there exists a vertex $z \in Z_{0} \operatorname{such}$ that $\operatorname{col}(z u) \neq c_{0}$. Then consider the theta graph $\left\{x v^{*}, x y v^{*}, x u z v^{*}\right\}$. We know that $\operatorname{col}(u z)=c_{i}$. However, this implies that $\left\{x v^{*}, x y v^{*}, x w u z v^{*}\right\}$ is a $\mathrm{PC} \Theta_{1,2,4}$, a contradiction. Thus $\operatorname{col}\left(Z_{0}, V(T)\right)=\left\{c_{0}\right\}$. For a vertex $z \in Z_{\beta}$, by considering the theta graphs $\left\{x z, x v^{*} z, x u z\right\}$ and $\left\{x z, x v^{*} z, x w u z\right\}$, we can obtain $\operatorname{col}(z u) \in\left\{c_{0}, \beta\right\}$. Thus $\operatorname{col}\left(Z_{\beta}, V(T)\right) \subseteq\left\{c_{0}, \beta\right\}$.

For each vertex $u \in\left(\cup_{i=1}^{p} S_{i} \backslash V(T)\right) \cup\left\{y, v^{*}\right\} \cup Z_{0} \cup Z_{\beta}$, by Subclaims 5.1 and 5.2, we have $N_{G^{\prime}}^{c}(u)=N_{G}^{c}(u)$. Thus $d_{G^{\prime}}^{c}(u)=d_{G}^{c}(u) \geq \delta^{c}(G)$. By Claims 1 and 2 , we know that $N_{G^{\prime}}^{c}(y) \backslash\{\beta\} \subseteq N_{G^{\prime}}^{c}(x) \backslash\{\alpha\}$. Thus $d_{G^{\prime}}^{c}(x) \geq d_{G^{\prime}}^{c}(y) \geq \delta^{c}(G)$. To obtain a final contradiction, it suffices to show that $d_{G^{\prime}}^{c}(z) \geq \delta^{c}(G)$ for each vertex $z \in Z_{\alpha}$.

Let $z$ be a vertex in $Z_{\alpha}$. If $N_{G^{\prime}}^{c}(y) \backslash\{\beta\} \subseteq N_{G^{\prime}}^{c}(z) \backslash\{\alpha\}$, then similarly, we can obtain $d_{G^{\prime}}^{c}(z) \geq d_{G^{\prime}}^{c}(y) \geq \delta^{c}(G)$. Now suppose that $N_{G^{\prime}}^{c}(y) \backslash\{\beta\} \nsubseteq N_{G^{\prime}}^{c}(z) \backslash\{\alpha\}$. Since $N_{G^{\prime}}^{c}(y) \backslash\{\beta\} \subseteq\left\{c_{i} \mid i \in[0, p]\right\}$ and $\operatorname{col}(x y)=\operatorname{col}(x z)=c_{0}$, there must exist a vertex $v_{i} \in S_{i} \backslash V(T)$ for some $i \in[1, p]$ such that $\operatorname{col}\left(y v_{i}\right)=c_{i}$ and $\operatorname{col}\left(z v_{i}\right) \neq c_{i}$. Note that if $\operatorname{col}(z, V(T)) \subseteq\left\{\alpha, c_{0}\right\}$, then $d_{G^{\prime}}^{c}(z)=d_{G}^{c}(z) \geq \delta^{c}(G)$. So we can assume that there exists a vertex $u_{j} \in S_{j} \cap V(T)$ for some $j \in[1, p]$ such that $\operatorname{col}\left(z u_{j}\right) \notin\left\{\alpha, c_{0}\right\}$. By Subclaim 5.1, we have $\operatorname{col}\left(y u_{j}\right)=c_{0}$. Recall that $\operatorname{col}\left(y v_{i}\right)=c_{i} \neq c_{0}$. So $v_{i} \neq u_{j}$. Considering the theta graph $\left\{z u_{j}, z x u_{j}, z v^{*} y u_{j}\right\}$, we get $\operatorname{col}\left(z u_{j}\right)=c_{j}$. Since $\left\{x v^{*} y, x u_{j} y, x z v_{i} y\right\}$ is not a PC theta graph and $\operatorname{col}\left(z v_{i}\right) \neq c_{i}$, we obtain 
that $\operatorname{col}\left(z v_{i}\right)=c_{0}$. However, this implies that $\left\{y v^{*} z, y u_{j} z, y v_{i} z\right\}$ is a $\mathrm{PC} \Theta_{2,2,2}$, a contradiction.

This completes the proof of Claim 5 .

Note that Subclaims 5.1 and 5.2, and the assertion that $S_{0}=Z_{0} \cup Z_{\alpha} \cup Z_{\beta}$ before Subclaim 5.2 are only valid in the proof of Claim 5. Now we continue the proof by three more claims.

Claim $6 d_{G-x}^{c}\left(v^{*}\right)=\delta^{c}(G)-1$, i.e., $d_{G}^{c}\left(v^{*}\right)=\delta^{c}(G)$ and $\alpha$ appears only once at $v^{*}$.

Proof Since $G$ is a CD-critical graph, we have $\delta^{c}(G-x)<\delta^{c}(G)$. Suppose, to the contrary, that $d_{G-x}^{c}\left(v^{*}\right) \geq \delta^{c}(G)$. Then by Claim 2, only vertices in $\{y\} \cup\left\{\cup_{i=1}^{p} S_{i}\right\}$ could suffer a color degree decrease to $\delta^{c}(G)-1$ when we delete the vertex $x$. If $d_{G-x}^{c}(y)=\delta^{c}(G)-1$, then by Claims 1 and 2 , we have $S_{0}=\varnothing$ and $\operatorname{col}\left(y, S_{i}\right)=c_{i}$ for all $i \in[1, p]$. So $d_{G-x}^{c}(y)=p+1$ and $\delta^{c}(G)=p+2$. For each vertex $u \in \cup_{i=1}^{p} S_{i}$, by Claims 4 and $5, N_{G}^{c}(u) \subseteq\left\{c_{i} \mid i \in[1, p]\right\} \cup\left\{\operatorname{col}\left(u v^{*}\right)\right\}$. Thus $d_{G}^{c}(u) \leq p+1<\delta^{c}(G)$, a contradiction. So $d_{G-x}^{c}(y) \geq \delta^{c}(y)$, and there must exist a vertex in $\cup_{i=1}^{p} S_{i}$, say a vertex $u_{1} \in S_{1}$, such that $d_{G-x}^{c}\left(u_{1}\right)=\delta^{c}(G)-1$. By Claims 1, 4 and 5, we have $\operatorname{col}\left(y u_{1}\right)=c_{0}, \operatorname{col}\left(v^{*} u_{1}\right) \in\{\alpha, \beta\}, \operatorname{col}\left(u_{1}, S_{i}\right)=\left\{c_{i}\right\}$ $(i \in[2, p])$ and $S_{1}=\left\{u_{1}\right\}$. Apply Claim 3 to $u_{1}$ and consider that $c_{1}$ appears only once at $u_{1}$. We have $\operatorname{col}\left(u_{1}, S_{0}\right) \subseteq\left\{c_{0}\right\}$. Now $N_{G}^{c}(y) \subseteq\left\{c_{i} \mid i \in[0, p], i \neq 1\right\} \cup\{\beta\}$ and $\delta^{c}(G) \leq d_{G}^{c}(y) \leq p+1$. Let $G^{\prime}=G-u_{1}$. Then $d_{G^{\prime}}^{c}(x)=p+2-1=p+1 \geq \delta^{c}(G)$ and for all $u \in V\left(G^{\prime}\right) \backslash\{x\}, d_{G^{\prime}}^{c}(u)=d_{G}^{c}(u) \geq \delta^{c}(G)$. So we have $\delta^{c}\left(G^{\prime}\right) \geq \delta^{c}(G)$, a contradiction.

Claim $7 \quad S_{0} \neq \emptyset$ and $d_{G-y}^{c}\left(v^{*}\right) \geq \delta^{c}(G)$.

Proof If $S_{0}=\emptyset$, then let $f\left(u_{i}\right)=c_{i}$ for all $u_{i} \in S_{i}$ and $i \in[1, p], f(x)=\alpha$, $f(y)=c_{0}$ and $f\left(v^{*}\right)=\beta$. By Claims $1,4,5$ and 6 , we have $\operatorname{col}(u v)=f(u)$ or $\operatorname{col}(u v)=f(v)$ for each edge $u v \in E(G)$, a contradiction. So $S_{0} \neq \varnothing$. Suppose that $d_{G-y}^{c}\left(v^{*}\right)=\delta^{c}(G)-1$. Then

$$
d_{G}^{c}\left(v^{*}\right)=\delta^{c}(G) \leq d_{G}^{c}(x)=p+2
$$

Together with Claim 6, we know that both colors $\alpha$ and $\beta$ appear only once at $v^{*}$. Thus, by Claim $1, \operatorname{col}\left(v^{*}, S_{i}\right)=\left\{c_{i}\right\}$ for all $i \in[1, p]$. This implies that $\beta \notin\left\{c_{i} \mid i \in[1, p]\right\}$ and

$$
d_{G}^{c}\left(v^{*}\right) \geq p+2
$$

So $d_{G}^{c}\left(v^{*}\right)=\delta^{c}(G)=p+2$ and $\operatorname{col}\left(v^{*}, S_{0}\right) \subseteq\left\{c_{i} \mid i \in[1, p]\right\}$. By applying Claim 3 to the vertex $v^{*}$, we know that $c_{0} \notin \operatorname{col}\left(y, \cup_{i=1}^{p} S_{i}\right)$. Thus $\operatorname{col}\left(\left\{x, y, v^{*}\right\}, S_{i}\right)$ $=\left\{c_{i}\right\}$ for all $i \in[1, p]$. Together with Claim 4, for each vertex $u \in \cup_{i=1}^{p} S_{i}$, we get $N_{G-S_{0}}^{c}(u) \subseteq\left\{c_{i} \mid i \in[1, p]\right\}$. Note that $\delta^{c}(G)=p+2$. So for a vertex $u_{1} \in S_{1}$, there must exist a vertex $z \in S_{0}$ such that $\operatorname{col}\left(z u_{1}\right) \notin\left\{c_{i} \mid i \in[0, p]\right\}$. This implies that $\left\{z v^{*}, z x v^{*}, z u_{1} y v^{*}\right\}$ is a $\mathrm{PC} \Theta_{1,2,3}$, a contradiction.

We need one more claim before we complete the proof of Lemma 3.1. 
Claim 8 The following statements hold:

(i) $\operatorname{col}\left(S_{0}, S_{i}\right) \subseteq\left\{c_{0}, c_{i}\right\}$ for all $i \in[1, p]$;

(ii) $\operatorname{col}\left(v^{*}, S_{j}\right) \subseteq\{\beta\} \cup\left\{c_{j}\right\}$ for all $j \in[0, p]$;

(iii) $\operatorname{col}\left(G\left[S_{0}\right]\right)=\left\{c_{0}\right\}$.

Proof Consider the graph $G-y$. Since $G$ is CD-critical, we have $\delta^{c}(G-y)<\delta^{c}(G)$. By Claims 2 and $7, d_{G-y}^{c}(x)=d_{G}^{c}(x) \geq \delta^{c}(G), d_{G-y}^{c}\left(v^{*}\right) \geq \delta^{c}(G)$ and for each vertex $z \in S_{0}, d_{G-y}^{c}(z)=d_{G}^{c}(z) \geq \delta^{c}(G)$. So there must exist a vertex $u^{*} \in \cup_{i=1}^{p} S_{i}$ such that $d_{G-y}^{c}\left(u^{*}\right)<\delta^{c}(G)$. Thus $\operatorname{col}\left(y u^{*}\right) \neq c_{i}$, i.e., $\operatorname{col}\left(y u^{*}\right)=c_{0}$ (by Claim 1). Apply Claim 3 to the edge $y u^{*}$. We get

$$
\operatorname{col}\left(S_{0}, S_{i}\right) \subseteq\left\{c_{0}, c_{i}\right\} \text { for all } i \in[1, p]
$$

and $\operatorname{col}\left(v^{*}, S_{0}\right) \subseteq\left\{\alpha, \beta, c_{0}\right\}$. By Claim $1, \operatorname{col}\left(v^{*}, S_{i}\right) \subseteq\{\alpha, \beta\} \cup\left\{c_{i}\right\}$ for all $i \in[1, p]$. So we have

$$
\operatorname{col}\left(v^{*}, S_{j}\right) \subseteq\{\alpha, \beta\} \cup\left\{c_{j}\right\} \text { for all } j \in[0, p]
$$

Considering Claim 6, we know that the color $\alpha$ appears only once at $v^{*}\left(\operatorname{col}\left(x v^{*}\right)=\alpha\right)$. Thus, in summary, Claim 8 ( $i$ ) and (ii) hold, and in particular, $S_{0}=Z_{0} \cup Z_{\beta}$. For vertices $z \in Z_{0}$ and $z^{\prime} \in S_{0}$ with $z \neq z^{\prime}$, since $\left\{x v^{*}, x u^{*} y v^{*}, x z^{\prime} z v^{*}\right\}$ is not a PC theta graph, we have $\operatorname{col}\left(z z^{\prime}\right)=c_{0}$. Thus $\operatorname{col}\left(G\left[Z_{0}\right]\right) \subseteq\left\{c_{0}\right\}$ and $\operatorname{col}\left(Z_{0}, Z_{\beta}\right)$ $\subseteq\left\{c_{0}\right\}$. To verify Claim 8 (iii), we are left to show that $\operatorname{col}\left(G\left[Z_{\beta}\right]\right) \subseteq\left\{c_{0}\right\}$. Let $T=\left\{u_{\beta} v_{\beta} \in E\left(G\left[Z_{\beta}\right]\right) \mid \operatorname{col}\left(u_{\beta} v_{\beta}\right) \neq c_{0}\right\}$. Suppose that $T \neq \emptyset$. Then let $G^{\prime}=G-V(T)$. Since $G$ is a CD-critical graph, there must exist a vertex $w \in V\left(G^{\prime}\right)$ such that $d_{G^{\prime}}^{c}(w)<\delta^{c}(G)$. Note that $V(T) \subseteq Z_{\beta}, \operatorname{col}\left(S_{0} \backslash V(T), V(T)\right) \subseteq\left\{c_{0}\right\}$, $\operatorname{col}(\{x, y\}, V(T))=\left\{c_{0}\right\}$ and $\operatorname{col}\left(v^{*}, V(T)\right)=\{\beta\}$. Therefore, for each vertex $u \in\left(S_{0} \backslash V(T)\right) \cup\left\{x, y, v^{*}\right\}$, we have $d_{G^{\prime}}^{c}(u)=d_{G}^{c}(u) \geq \delta^{c}(G)$. This implies that $w \in S_{j}$ for some $j \in[1, p]$. By Claim $8(i), \operatorname{col}(w, V(T))$ $\subseteq\left\{c_{0}, c_{j}\right\}$. This forces $N_{G}^{c}(w) \backslash N_{G^{\prime}}^{c}(w)=\left\{c_{0}\right\}$ by the fact that $\operatorname{col}(x w)=c_{j}$. Thus $\operatorname{col}(y w)=c_{j}$ and there exists an edge $u_{\beta} v_{\beta} \in T$ such that $\operatorname{col}\left(u_{\beta} w\right)=c_{0}$. Recall that $\operatorname{col}\left(y u^{*}\right)=c_{0}$. We obtain that $u^{*} \neq w$ and that $\left\{x v^{*} y, x u^{*} y, x v_{\beta} u \beta w y\right\}$ is a PC $\Theta_{2,2,4}$, a contradiction. So, $\operatorname{col}\left(G\left[Z_{\beta}\right]\right) \subseteq\left\{c_{0}\right\}$. This completes the proof of Claim 8 .

Now define a function $f$ such that $f(x)=\alpha, f(y)=c_{0}, f\left(v^{*}\right)=\beta$ and $f\left(u_{i}\right)=c_{i}$ for each vertex $u_{i} \in S_{i}$ and $i \in[0, p]$. By Claims 1, 4, 5 and 8, we conclude that $\operatorname{col}(u v)=f(u)$ or $\operatorname{col}(u v)=f(v)$ for each edge $u v \in E(G)$, a contradiction. This completes the proof of Lemma 3.1.

Before presenting the proof of Lemma 3.2, we need the following observation.

Observation 3.3 (Fujita et al. [6]) Let $G$ be a colored complete bipartite graph. If $\delta^{c}(G) \geq 2$, then $G$ contains a PC cycle of length 4 or 6 .

Proof of Lemma 3.2 By contradiction. Suppose, to the contrary, that there exists an edge $x y \in E(G)$ such that $d_{G-y}^{c}(x)=\delta^{c}(G)-1$ and $d_{G-x}^{c}(y)=\delta^{c}(G)-1$. Then $x \in \operatorname{Dom}_{G}(y), y \in \operatorname{Dom}_{G}(x)$ and $d^{c}(x)=d^{c}(y)=\delta^{c}(G)$. Let $\alpha=\operatorname{col}(x y)$. Define 


$$
S=\{u \in V(G) \backslash\{x, y\} \mid \operatorname{col}(u x) \neq \operatorname{col}(u y)\}
$$

and

$$
T=\{u \in V(G) \backslash\{x, y\} \mid \operatorname{col}(u x)=\operatorname{col}(u y)\} .
$$

Then $V(G)=S \cup T \cup\{x, y\}$. Since $\left|\operatorname{col}\left(x, \operatorname{Dom}_{G}(x)\right)\right|=\left|\operatorname{col}\left(y, \operatorname{Dom}_{G}(y)\right)\right|=1$, we know that $\operatorname{col}\left(x, \operatorname{Dom}_{G}(x)\right)=\operatorname{col}\left(y, \operatorname{Dom}_{G}(y)\right)=\{\alpha\}$ and the color $\alpha$ appears only once at $x$ and $y$. Thus $\operatorname{Dom}_{G}(x)=\{y\}, \operatorname{Dom}_{G}(y)=\{x\}$ and for each vertex $u \in S$, both colors $\operatorname{col}(x u)$ and $\operatorname{col}(y u)$ appear at least twice at $u$. So we have $d_{G-\{x, y\}}^{c}(u)=d_{G}^{c}(u)$. Noting that $\delta^{c}(G-\{x, y\})<\delta^{c}(G)$, there must exist a vertex $z \in T$ such that $d_{G-\{x, y\}}^{c}(z)<\delta^{c}(G)$. Let $\operatorname{col}(z x)=\operatorname{col}(z y)=\beta$. Then $\beta \notin \operatorname{col}(z, V(G) \backslash\{x, y, z\})$. Throughout the proof, the notations $x, y, z, \alpha$ and $\beta$ always refer to the vertices and colors stated above. Now we deliver the proof by first proving the following claims.

Claim $1 S=\emptyset$.

Proof Suppose, to the contrary, that $S \neq \emptyset$. Let $H$ be the complete bipartite subgraph of $G$ with partite sets $\{x, y\}$ and $S$. If $\delta^{c}(H) \geq 2$, then by Observation 3.3, $H$ must contain a PC cycle passing through vertices $x$ and $y$ (which are not consecutive on this cycle). Combining this cycle with the edge $x y$, we obtain a PC theta graph in $G$, a contradiction. So, we have $\delta^{c}(H)=1$. Note that for all $u \in S, d_{H}^{c}(u) \geq 2$. Hence, $d_{H}^{c}(x)=1$ or $d_{H}^{c}(y)=1$. Without loss of generality, assume that $d_{H}^{c}(x)=1$ and $\operatorname{col}(x, S)=\left\{c_{0}\right\}$.

Subclaim 1.1 For each pair of vertices $v_{1}, v_{2} \in T$ with $\operatorname{col}\left(v_{1} x\right) \neq \operatorname{col}\left(v_{2} x\right)$, we have $\operatorname{col}\left(v_{1} v_{2}\right) \in\left\{\operatorname{col}\left(v_{1} x\right), \operatorname{col}\left(v_{2} x\right)\right\}$.

Proof Suppose, to the contrary, that $\operatorname{col}\left(v_{1} v_{2}\right) \notin\left\{\operatorname{col}\left(v_{1} x\right), \operatorname{col}\left(v_{2} x\right)\right\}$ for some vertices $v_{1}, v_{2} \in T$ satisfying $\operatorname{col}\left(v_{1} x\right) \neq \operatorname{col}\left(v_{2} x\right)$. Choose a vertex $u \in S$. Then $\operatorname{col}(u x)=c_{0}$ and $\operatorname{col}(u y) \neq c_{0}$. Let $\epsilon=\operatorname{col}(u y)$. Then $\epsilon \neq c_{0}$. Now consider the colors of $v_{1} x$ and $v_{2} y$. If $\operatorname{col}\left(v_{1} x\right)=c_{0}$, then $\operatorname{col}\left(v_{1} y\right)=\operatorname{col}\left(v_{1} x\right)=c_{0}$ and $\operatorname{col}\left(v_{2} x\right) \neq c_{0}$. This implies that $\left\{x y, x u y, x v_{2} v_{1} y\right\}$ is a PC $\Theta_{1,2,3}$, a contradiction. If $\operatorname{col}\left(v_{2} y\right)=\epsilon$, then $\operatorname{col}\left(v_{2} x\right)=\operatorname{col}\left(v_{2} y\right)=\epsilon$ and $\operatorname{col}\left(v_{1} y\right) \neq \epsilon$. This again implies that $\left\{x y, x u y, x v_{2} v_{1} y\right\}$ is a PC $\Theta_{1,2,3}$, a contradiction. So we have $\operatorname{col}\left(v_{1} x\right) \neq c_{0}$ and $\operatorname{col}\left(v_{2} y\right) \neq \epsilon$. However, this forces $\left\{x y, x u y, x v_{1} v_{2} y\right\}$ to be a PC $\Theta_{1,2,3}$, a contradiction.

Subclaim 1.2 Either $\operatorname{col}(z u)=\operatorname{col}(y u)$ for all $u \in S$ or $\operatorname{col}(z, S)=\left\{c_{0}\right\}$.

Proof It is equivalent to show that $\operatorname{col}(z u) \in\left\{c_{0}, \operatorname{col}(y u)\right\}$ for each vertex $u \in S$, and there cannot exist two vertices $u_{1}, u_{2} \in S$ such that $\operatorname{col}\left(z u_{1}\right)$ $=c_{0}$ and $\operatorname{col}\left(z u_{2}\right)=\operatorname{col}\left(y u_{2}\right)$.

Let $u$ be a vertex in $S$. Since $\beta \notin \operatorname{col}(z, V(G) \backslash\{x, y, z\})$, we have $\operatorname{col}(z u)$ $\neq \beta$. Suppose that $\operatorname{col}(z u) \notin\left\{c_{0}, \operatorname{col}(u y)\right\}$. Then consider the relation between $c_{0}$ and $\beta$. If $c_{0} \neq \beta$, then $\{x u, x y u, x z u\}$ is a $\mathrm{PC} \Theta_{1,2,2}$, a contradiction. If $c_{0}=\beta$, then $\operatorname{col}(y u) \neq \beta$ and $\{y u, y x u, y z u\}$ is a PC $\Theta_{1,2,2}$, a contradiction. So 
$\operatorname{col}(z u) \in\left\{c_{0}, \operatorname{col}(u y)\right\}$. Now suppose that there are two vertices $u_{1}, u_{2} \in S$ such that $\operatorname{col}\left(z u_{1}\right)=c_{0}$ and $\operatorname{col}\left(z u_{2}\right)=\operatorname{col}\left(y u_{2}\right)$. Since $\beta \notin \operatorname{col}(z, V(G) \backslash\{x, y, z\})$, we obtain $c_{0} \neq \beta$ and $\operatorname{col}\left(z u_{2}\right) \neq \beta$. Noting that $\operatorname{col}\left(x u_{2}\right)=c_{0}$, we obtain a PC theta graph $\left\{z x, z u_{2} x, z u_{1} y x\right\}$, a contradiction.

Subclaim 1.3 The following statements hold:

(i) there exists a vertex $z^{\prime} \in T-z$ such that $\operatorname{col}\left(x z^{\prime}\right)=\beta$;

(ii) either $c_{0}=\beta$ or $\operatorname{col}(y, S)=\beta$.

Proof Consider the colors appearing on the edges incident with $z$. If $\beta \notin \operatorname{col}(T-z,\{x, y\})$, then apply Subclaim 1.1 to $z$ and each vertex in $T-z$. Recall that $\beta \notin \operatorname{col}(z, V(G) \backslash\{x, y, z\})$. We obtain

$$
\operatorname{col}(z, T-z)=\operatorname{col}(x, T-z)=\operatorname{col}(y, T-z) .
$$

Using Subclaim 1.2, we conclude that either

$$
N^{c}(z)=\operatorname{col}(x, T) \cup\left\{c_{0}\right\}=N^{c}(x) \backslash\{\alpha\}
$$

or

$$
N^{c}(z)=\operatorname{col}(y, T) \cup \operatorname{col}(y, S)=N^{c}(y) \backslash\{\alpha\} .
$$

Since $d^{c}(x)=d^{c}(y)=\delta^{c}(G)$, we have $d^{c}(z)=\delta^{c}(G)-1$, a contradiction. So there must exist a vertex $z^{\prime} \in T$ with $z^{\prime} \neq z$ such that $\operatorname{col}\left(z^{\prime} x\right)=\operatorname{col}\left(z^{\prime} y\right)=\beta$. Let $\epsilon=\operatorname{col}\left(z z^{\prime}\right)$. Recall that $\beta \notin \operatorname{col}(z, V(G) \backslash\{x, y, z\})$. We obtain that $\epsilon \neq \beta$. For a vertex $u \in S$, since $\left\{x y, x z z^{\prime} y, x u y\right\}$ is not a PC theta graph, we have $c_{0}=\beta$ or $\operatorname{col}(y u)=\beta$. This implies that either $c_{0}=\beta$ or $\operatorname{col}(y, S)=\beta$.

Now we need another vertex in $T$ to continue the proof of Claim 1. If $\operatorname{col}(x, T)$ $=\{\beta\}$, then $\operatorname{col}(y, T)=\operatorname{col}(x, T)=\beta$. Together with Subclaim 1.3 (ii), we obtain that either $N^{c}(x)=\{\alpha, \beta\}$ or $N^{c}(y)=\{\alpha, \beta\}$. This contradicts that $\delta^{c}(G) \geq 3$. Hence there must exist a vertex $w \in T$ such that $\operatorname{col}(w x) \neq \beta$. Let $\lambda=\operatorname{col}(w x)$ and let $z^{\prime}$ be the vertex in Subclaim $1.3(i)$. If there exists a vertex $w^{\prime} \in T$ satisfying $\operatorname{col}\left(w^{\prime} x\right)=\lambda$ and $\operatorname{col}\left(w w^{\prime}\right) \neq \lambda$, then $\left\{x y, x z z^{\prime} y, x w w^{\prime} y\right\}$ is a PC $\Theta_{1,3,3}$, a contradiction. So we have $\operatorname{col}(w v)=\lambda$ for all vertices $v \in T$ satisfying $\operatorname{col}(v x)=\lambda$. Together with Subclaim 1.1, we obtain $\operatorname{col}(w v) \in\{\lambda\} \cup\{\operatorname{col}(v x)\}$ for all $v \in T-w$. Thus

$$
\operatorname{col}(w, T-w) \subseteq \operatorname{col}(\{x, y\}, T)
$$

Now we will complete the proof of Claim 1 by considering the two cases for $c_{0}$ and $\beta$. If $c_{0}=\beta$, then $\beta \notin \operatorname{col}(y, S)$ and for each vertex $u \in S, \operatorname{col}(u w) \in\{\lambda\} \cup\{\operatorname{col}(u y)\}$ (otherwise $\left\{x y, x z z^{\prime} y, x w u y\right\}$ is a PC theta graph). This implies that $\operatorname{col}(w, S)$ $\subseteq \operatorname{col}(y, S) \cup\{\lambda\}$. Thus

$$
N_{G}^{c}(w)=\operatorname{col}(w, T-w) \cup \operatorname{col}(w, S) \cup\{\lambda\} \subseteq N_{G}^{c}(y) \backslash\{\alpha\}
$$


So we have $d_{G}^{c}(w) \leq d_{G}^{c}(y)-1<\delta^{c}(G)$, a contradiction. If $c_{0} \neq \beta$, then for each vertex $u \in S$, either $\operatorname{col}(u w)=\lambda$ or $\operatorname{col}(u w)=c_{0}$ (otherwise $\left\{x y, x z z^{\prime} y, x u w y\right\}$ is a PC theta graph). This implies that $\operatorname{col}(w, S) \subseteq \operatorname{col}(x, S) \cup\{\lambda\}$. Thus

$$
N_{G}^{c}(w)=\operatorname{col}(w, T-w) \cup \operatorname{col}(w, S) \cup\{\lambda\} \subseteq N_{G}^{c}(x) \backslash\{\alpha\} .
$$

So we have $d_{G}^{c}(w) \leq d_{G}^{c}(x)-1<\delta^{c}(G)$, a contradiction. This completes the proof of Claim 1.

Since $S=\emptyset$, the use of $c_{0}$ in the above proof of Claim 1 is not valid anymore in the remainder of the proof. We can assume that $N^{c}(x)=\left\{\alpha, \beta, c_{1}, c_{2}, \ldots, c_{p}\right\}$ with $p \geq 1\left(\right.$ since $\left.\delta^{c}(G) \geq 3\right)$. Let $T_{i}=\left\{v \in T \mid \operatorname{col}(v x)=c_{i}\right\}$ for all $i \in[1, p]$. Let $T_{\beta}=\{v \in T \mid \operatorname{col}(v x)=\beta\}$. Then $V(G)=\left(\cup_{i=1}^{p} T_{i}\right) \cup T_{\beta} \cup\{x, y\}$ and $\left|\cup_{i=1}^{p} T_{i}\right| \geq 1$. We need one additional claim.

Claim $2 T_{\beta}=\{z\}$.

Proof Suppose the contrary. Then for each vertex $w \in T_{\beta}-z, \operatorname{col}(z w) \neq \beta$ (since $\beta \notin \operatorname{col}(z, V(G) \backslash\{x, y, z\}))$. Consider the cardinality of $\cup_{i=1}^{p} T_{i}$.

If $\left|\cup_{i=1}^{p} T_{i}\right|=1$, then let $u$ be the unique vertex in $\cup_{i=1}^{p} T_{i}$ and let $\operatorname{col}(\{x, y\}, u)$ $=\left\{c_{1}\right\}$. Thus $V(G)=T_{\beta} \cup\{x, y, u\}$. Since $d^{c}(u) \geq 3$, there exists a vertex $w \in T_{\beta}$ such that $\operatorname{col}(u w) \notin\left\{c_{1}, \beta\right\}$. Furthermore, since $d^{c}(w) \geq 3$, there exists a vertex $w^{\prime} \in T_{\beta}-w$ such that $\operatorname{col}\left(w w^{\prime}\right) \notin\{\beta, \operatorname{col}(u w)\}$. Now $\left\{x y w, x u w, x w^{\prime} w\right\}$ is a PC $\Theta_{2,2,2}$, a contradiction. Now consider $\left|\cup_{i=1}^{p} T_{i}\right| \geq 2$. By the assumption that $\left|T_{\beta}\right| \geq 2$, we can choose a vertex $w \in T_{\beta} \backslash\{z\}$. For every pair of distinct vertices $u, v \in \cup_{i=1}^{p} T_{i}$, since $\{x y, x z w y, x u v y\}$ is not a PC theta graph, we have $\operatorname{col}(u v)=\operatorname{col}(u x)$ or $\operatorname{col}(u v)=\operatorname{col}(v x)$. This implies that $\operatorname{col}\left(G\left[\cup_{i=1}^{p} T_{i}\right]\right)$ $\subseteq\left\{c_{1}, c_{2}, \ldots, c_{p}\right\}$. Let $u, v \in \cup_{i=1}^{p} T_{i}$ be arbitrarily chosen distinct vertices. Note that $d_{G}^{c}(u), d_{G}^{c}(v) \geq \delta^{c}(G)$ and $\delta^{c}(G)=d^{c}(x)=p+2$. There must exist vertices $z_{u}, z_{v} \in T_{\beta}$ such that $\operatorname{col}\left(u z_{u}\right), \operatorname{col}\left(v z_{v}\right) \notin\left\{\beta, c_{1}, c_{2}, \ldots, c_{p}\right\}$. If $z_{u} \neq z_{v}$, then $\left\{x y, x u z_{u} y, x z_{v} v y\right\}$ is a PC $\Theta_{1,3,3}$, a contradiction. So there must exist a vertex $z^{*} \in T_{\beta}$ such that $\operatorname{col}\left(\cup_{i=1}^{p} T_{i}, z^{*}\right) \cap\left\{\beta, c_{1}, c_{2}, \ldots, c_{p}\right\}=\emptyset$ and $\operatorname{col}\left(\cup_{i=1}^{p} T_{i}, T_{\beta}-z^{*}\right)$ $\subseteq\left\{\beta, c_{1}, c_{2}, \ldots, c_{p}\right\}$. Thus $\operatorname{col}\left(u z^{*}\right)$ appears only once at $u$ for each vertex $u \in \cup_{i=1}^{p} T_{i}$, i.e., $\cup_{i=1}^{p} T_{i} \subseteq \operatorname{Dom}_{G}\left(z^{*}\right)$. Note that $\left|\operatorname{col}\left(z^{*}, \operatorname{Dom}_{G}\left(z^{*}\right)\right)\right|=1$. We can assume that $\operatorname{col}\left(z^{*}, \cup_{i=1}^{p} T_{i}\right)=\{\eta\}$. Then $\eta \notin\left\{\beta, c_{1}, c_{2}, \ldots, c_{p}\right\}$. Since $d_{G}^{c}\left(z^{*}\right) \geq 3$, there must exist a vertex $z^{\prime} \in T_{\beta}-z^{*}$ such that $\operatorname{col}\left(z^{*} z^{\prime}\right) \notin\{\beta, \eta\}$. Choose a vertex $u \in \cup_{i=1}^{p} T_{i}$. Then $\left\{z^{*} u x, z^{*} y x, z^{*} z^{\prime} x\right\}$ is a PC $\Theta_{2,2,2}$, a contradiction.

Claims 1 and 2 imply that $\{x, y, z\}, T_{1}, T_{2}, \ldots, T_{p}$ form a partition of $G$. Since $\delta^{c}(G)=d^{c}(x)=p+2$, there exists a pair of vertices $u, u^{\prime} \in \cup_{i=1}^{p} T_{i}$ such that $\operatorname{col}(z u) \notin\left\{\beta, c_{1}, c_{2}, \ldots, c_{p}\right\}$ and $\operatorname{col}\left(u u^{\prime}\right) \notin\left\{\operatorname{col}(z u), c_{1}, c_{2}, \ldots, c_{p}\right\}$. This implies that $\left\{x u, x z u, x y u^{\prime} u\right\}$ is a PC $\Theta_{1,2,3}$, a contradiction. This completes the proof of Lemma 3.2.

\section{PC Theta Graphs of Small Order}

In [4], PC $K_{4}$ 's are used to merge vertices into a PC Hamilton cycle. For the existence of a PC $K_{4}$, the existence of a PC $\Theta_{1,2,2}$ is clearly necessary. In this section, we give 
a color number condition for the existence of small PC theta graphs. The proof of the following result is inspired by the proof of Lemma 4.1 in [20].

Theorem 4.1 Let $G$ be a colored $K_{n}$. If $|\operatorname{col}(G)| \geq n+1$, then $G$ contains a $P C \Theta_{1,2,2}$ or a $P C \Theta_{1,2,3}$.

Remark 4.1 ( $i$ ) The bound " $n+1$ " in Theorem 4.1 is tight in the following sense. Let $T$ be a tournament with $V(T)=\left\{u_{i} \mid i \in[1, n]\right\}$ and $\delta^{+}(T) \geq 1$. Let $G$ be a colored $K_{n}$ with $V(G)=V(T)$ and $\operatorname{col}\left(u_{i} u_{j}\right)=i$ if $u_{i} u_{j} \in A(T)$. Then $|\operatorname{col}(G)|=n$ and $G$ contains no PC theta graph.

(ii) The bound " $n+1$ " in Theorem 4.1 cannot guarantee the existence of a PC $\Theta_{1,2,2}$. Given an integer $k \geq 3$, let $G$ be a colored graph such that $V(G)=\{u\}$ $\cup\left\{v_{1}, v_{2}, v_{3}\right\} \cup\left\{w_{1}, w_{2}, \ldots, w_{k}\right\}, \operatorname{col}\left(u w_{i}\right)=c_{i}$ for $i \in[1, k], \operatorname{col}\left(w_{i} w_{j}\right)=\alpha$ for all $i, j \in[1, p]$ with $i<j, \operatorname{col}\left(\left\{v_{1}, v_{2}, v_{3}\right\},\left\{u, w_{1}, w_{2}, \ldots, w_{k}\right\}\right)=\beta$, and $v_{1} v_{2} v_{3} v_{1}$ is a PC triangle with three new colors. Then $G$ is a colored complete graph with $|\operatorname{col}(G)|=|V(G)|+1$. But $G$ contains no PC $\Theta_{1,2,2}$.

(iii) The bound " $n+1$ " in Theorem 4.1 cannot guarantee the existence of a PC $\Theta_{1,2,3}$. Let $G_{1}$ be a colored $K_{4}$ with all the edges in different colors. For $i \geq 1$, construct $G_{i+1}$ by joining $G_{i}$ and a PC triangle $T_{i}=x_{i} y_{i} z_{i} x_{i}$ with $\operatorname{col}\left(G_{i}\right) \cap \operatorname{col}\left(T_{i}\right)=\emptyset, \operatorname{col}\left(x_{i}, G_{i}\right)=\left\{\operatorname{col}\left(x_{i} y_{i}\right)\right\}, \operatorname{col}\left(y_{i}, G_{i}\right)=\left\{\operatorname{col}\left(y_{i} z_{i}\right)\right\}$ and $\operatorname{col}\left(z_{i}, G_{i}\right)=\left\{\operatorname{col}\left(z_{i} x_{i}\right)\right\}$. Then $\left|\operatorname{col}\left(G_{i}\right)\right|=\left|V\left(G_{i}\right)\right|+2$ for all $i \geq 1$. But $G_{i}$ contains no $\mathrm{PC} \Theta_{1,2,3}$.

Proof of Theorem 4.1 By contradiction. Let $G \cong K_{n}$ be a counterexample to Theorem 4.1 such that $n$ is as small as possible, and subject to this, $|\operatorname{col}(G)|$ is as small as possible. Obviously, $n \geq 5,|\operatorname{col}(G)|=n+1$ (otherwise, by merging two colors into a new color, we obtain a counterexample to Theorem 4.1 with a smaller number of colors) and $|\operatorname{col}(G-S)| \leq n-|S|$ for each nonempty subset $S$ of $V(G)$. In particular, when $S$ is a single vertex $v$, we have $|\operatorname{col}(G)|-|\operatorname{col}(G-v)| \geq 2$. For each vertex $v \in V(G)$, define $d_{G}^{s}(v)=|\operatorname{col}(G)|-|\operatorname{col}(G-v)|$. Let

$$
E^{*}=\{u v \in E(G) \mid \operatorname{col}(u v) \notin \operatorname{col}(G-u v)\} .
$$

Then we have

$$
2\left|E^{*}\right|+\left(|\operatorname{col}(G)|-\left|E^{*}\right|\right) \geq \sum_{v \in V(G)} d_{G}^{s}(v) \geq 2 n .
$$

Thus $\left|E^{*}\right| \geq n-1$. Let $H$ be the subgraph of $G$ induced by $E^{*}$. Then there must exist a vertex $x \in V(H)$ and an integer $k \in[2, n-1]$ such that $d_{H}(x)=k($ since $n-1>n / 2)$. Let $u_{1}, u_{2}, \ldots, u_{k}$ be the neighbors of $x$ in $H$. Let $G_{1}=G\left[\left\{u_{1}, u_{2}, \ldots, u_{k}, x\right\}\right]$ and $G_{2}=G-G_{1}$. Then $G_{1}-x$ is a monochromatic complete graph (otherwise, there exist vertices $u, v, w \in V\left(G_{1}\right) \backslash\{x\}$ such that $\operatorname{col}(u v) \neq \operatorname{col}(u w)$ and $\{x u, x v u, x w u\}$ is a $\left.\mathrm{PC} \Theta_{1,2,2}\right)$. Thus $\left|\operatorname{col}\left(G_{1}\right)\right|=k+1<n+1$ and $G_{2}$ is nonempty. We claim that $E\left(G_{1}, G_{2}\right) \cap E^{*}=\emptyset$. For each edge $u v \in E\left(G_{1}, G_{2}\right)$ with $u \in V\left(G_{1}\right)$ and $v \in V\left(G_{2}\right)$, if $u=x$, then by the construction of $G_{1}$, we know that $u v \notin E^{*}$; otherwise, $u \in V\left(G_{1}\right) \backslash\{x\}$. Then, choose a vertex $u^{\prime} \in V\left(G_{1}\right) \backslash\{x, u\}$ (this is 
doable because $k \geq 2$ ). Since $\left\{x u, x u^{\prime} u, x v u\right\}$ is not a PC theta graph, we have $\operatorname{col}(u v) \in\left\{\operatorname{col}\left(u u^{\prime}\right), \operatorname{col}(x v)\right\}$, and, in particular $u v \notin E^{*}$.

We will complete the proof by distinguishing the following two cases.

Case $1 u_{1} u_{2} \in E^{*}$.

In this case, the color $\operatorname{col}\left(u_{1} u_{2}\right)$ appears only once on $E(G)$. Note that $G_{1}-x$ is a monochromatic complete graph. We have $k=2$ and $\left|E^{*}\right| \geq n-1>3=\left|E\left(G_{1}\right)\right|$. Since $E\left(G_{1}, G_{2}\right) \cap E^{*}=\emptyset$, there must exist an edge $y z \in E\left(G_{2}\right) \cap E^{*}$. This implies that $\left\{x u_{1}, x u_{2} u_{1}, x y z u_{1}\right\}$ is a PC $\Theta_{1,2,3}$, a contradiction.

Case $2 u_{1} u_{2} \notin E^{*}$.

In this case, the only color in $\operatorname{col}\left(G_{1}-x\right)$ does not appear on $E^{*}$. Thus $E\left(G_{1}\right) \cap E^{*}$ $=\left\{x u_{i} \mid i \in[1, k]\right\}$. Recall that $E\left(G_{1}, G_{2}\right) \cap E^{*}=\emptyset$ and $\left|E^{*}\right| \geq n-1$. We have

$$
\left|\operatorname{col}\left(G_{2}\right)\right| \geq\left|E\left(G_{2}\right) \cap E^{*}\right| \geq n-k-1 .
$$

Note that $\left|\operatorname{col}\left(G_{2}\right)\right| \leq n-\left|G_{1}\right|=n-k-1$. We conclude that $\left|\operatorname{col}\left(G_{2}\right)\right|=n-k-1$ and that all inequalities in (1) are equalities. Thus by the definition of $E^{*}$, we have $E\left(G_{2}\right) \subseteq E^{*}$ and $\left|E\left(G_{2}\right)\right|=n-k-1=\left|V\left(G_{2}\right)\right|$. This forces $G_{2}$ to be a PC triangle with all its edges contained in $E^{*}$. Let $G_{2}=y z w y$. Then $\left\{y z, y w z, y x u_{1} z\right\}$ is a PC $\Theta_{1,2,3}$, a contradiction.

The proof of Theorem 4.1 is complete.

\section{PC Theta Graphs of Large Order}

In this section, we present a sufficient color degree condition for the existence of large PC theta graphs in colored complete graphs. Our main result of this section is related to the existence of long PC cycles in colored complete graphs, and in particular to the following conjecture due to Fujita and Magnant [7].

Conjecture 5.1 (Fujita and Magnant [7]) Let $G$ be a colored $K_{n}$. If $\delta^{c}(G) \geq \frac{n+1}{2}$, then each vertex of $G$ is contained in a PC cycle of length $\ell$ for all $\ell \in[3, n]$.

In the same paper, they presented a class of colored complete graphs to show that the statement of Conjecture 5.1 would be best possible (the lower bound on $\delta^{c}(G)$ cannot be improved), and they proved that each vertex is contained in a PC cycle of length 3 , 4 , and when $n \geq 13$, also in a PC cycle of length 5. Recently, Lo [19] established the existence of a PC Hamilton cycle (a PC cycle of length $n$ ) in $G$ for sufficiently large $n$ when $\delta^{c}(G) \geq(1 / 2+\epsilon) n$ for any arbitrarily small constant $\epsilon>0$. In this context, in [15] the following cycle extension theorem was proved.

Theorem 5.2 (Li et al. [15]) Let $G$ be a colored $K_{n}$ and let $C$ be a PC cycle of length $k$ in $G$. If $\delta^{c}(G) \geq \max \left\{\frac{n-k}{2}, k\right\}+1$, then $G$ contains a PC cycle $C^{*}$ such that $V(C) \subset V\left(C^{*}\right)$ and $\left|C^{*}\right|>k$. 
Using the result of [7] that each vertex is contained in a small PC cycle, as a corollary of Theorem 5.2, it is easy to obtain the following result.

Corollary 5.3 (Li et al. [15]) Let $G$ be a colored $K_{n}$. If $\delta^{c}(G) \geq \frac{n+1}{2}$, then each vertex of $G$ is contained in a PC cycle of length at least $\delta^{c}(G)$.

Interestingly, under the condition $\delta^{c}(G) \geq \frac{n+1}{2}$ of Conjecture 5.1, even the existence of a PC Hamilton cycle has not been fully verified.

In the remainder of this section, we discuss the existence of PC theta graphs when $\delta^{c}(G) \geq \frac{n+1}{2}$. We first need the following natural definition of a maximal PC cycle.

Definition 5.1 A PC cycle $C$ in $G$ is called a maximal PC cycle if there is no longer PC cycle $C^{\prime}$ in $G$ with $V(C) \subset V\left(C^{\prime}\right)$.

Now we are ready to present the main result of this section.

Theorem 5.4 Let $G$ be a colored $K_{n}$. If $\delta^{c}(G) \geq \frac{n+1}{2}$, then one of the following statements holds:

(i) $d^{c}(u)=\frac{n+1}{2}$ for each vertex $u \in V(G)$ and $G$ contains a PC Hamilton cycle;

(ii) each maximal PC cycle $C$ in $G$ has a chord $u v$ such that $\left\{u v, u C^{+} v, u C^{-} v\right\}$ is a PC theta graph.

Before we give our proof of the above theorem, as a final result of this section we present the following straightforward corollary of Theorem 5.4 and Corollary 5.3 without a proof.

Corollary 5.5 Let $G$ be a colored $K_{n}$. If $\delta^{c}(G) \geq \frac{n}{2}+1$, then each vertex of $G$ is contained in a PC theta graph $\Theta_{1, k, m}$ such that $k+m \geq \delta^{c}(G)$.

We use the following lemma in our proof of Theorem 5.4.

Lemma 5.6 Let $G$ be a colored $K_{n}$ and let $C=v_{1} v_{2} \ldots v_{\ell} v_{1}$ be a PC cycle in $G$. If there exists a PC path $P=u_{0} u_{1} \ldots u_{p}(p \geq 1)$ in $G$ such that $V(P) \cap V(C)$ $=\left\{u_{p}\right\}=\left\{v_{i}\right\}$ for some $i \in[1, \ell]$ and $\operatorname{col}\left(u_{p-1} u_{p}\right) \notin\left\{\operatorname{col}\left(v_{i-1} v_{i}\right), \operatorname{col}\left(v_{i} v_{i+1}\right)\right\}$ (where indices of $v_{i}$ are taken modulo $\ell$ ), then for each vertex $v \in V(G) \backslash(V(P) \cup$ $V(C)$ ) there exists a PC path $Q=u_{0} w_{1} w_{2} \ldots w_{q} v$ such that $V(C) \subset V(Q), w_{q}$ $\in V(C)$ and $w_{1} \in V(C) \cup\left\{u_{1}\right\}$.

Proof Suppose, to the contrary, that there does not exist such a PC path. Without loss of generality, assume that $i=1$. If $\operatorname{col}\left(v v_{2}\right) \neq \operatorname{col}\left(v_{2} v_{3}\right)$, then $u_{0} P v_{1} v_{\ell} v_{\ell-1} \cdots v_{2} v$ is a PC path satisfying the statement in Lemma 5.6, a contradiction. So we have $\operatorname{col}\left(v v_{2}\right)=\operatorname{col}\left(v_{2} v_{3}\right)$. Since $v v_{2} v_{1} v_{\ell} \cdots v_{3} u_{0}$ is not a PC path, we get $\operatorname{col}\left(u_{0} v_{3}\right)$ $=\operatorname{col}\left(v_{3} v_{4}\right)$. Repeating these arguments, switching between $u_{0}$ and $v$, if $\ell$ is even, we obtain $\operatorname{col}\left(v v_{\ell}\right)=\operatorname{col}\left(v_{\ell} v_{1}\right)$ after going along $C$ in one round; if $\ell$ is odd, we obtain $\operatorname{col}\left(v v_{\ell}\right)=\operatorname{col}\left(v_{\ell} v_{1}\right)$ after two rounds. In both cases, we end up with a PC path $u_{0} P v_{1} v_{2} \cdots v_{\ell} v$ satisfying the statement in Lemma 5.6, a contradiction.

Now we present our proof of Theorem 5.4.

Proof of Theorem 5.4 Let $G$ be a colored $K_{n}$ satisfying $\delta^{c}(G) \geq \frac{n+1}{2}$ and suppose that $C=v_{1} v_{2} \ldots v_{\ell} v_{1}$ is a maximal PC cycle in $G$ for which statement $(i i)$ of the theorem does not hold. Then, by Theorem 5.2 we know that $\ell \geq \delta^{c}(G)$. Now we generate a set of subgraphs of $G$ using Algorithm 1. 


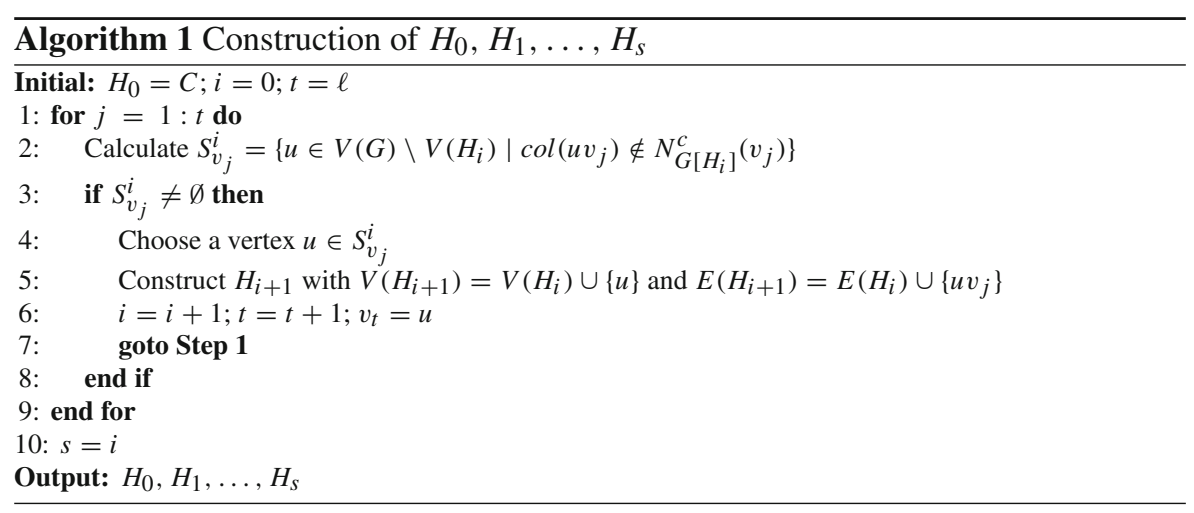

Fig. 6 The structure of $H_{S}$ obtained from Algorithm 1

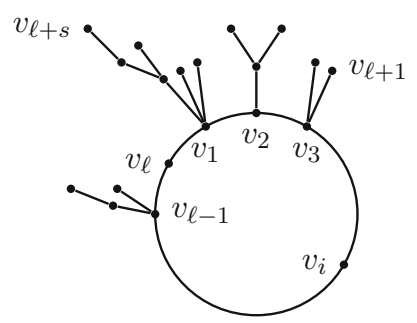

In Algorithm 1, $H_{0}=C$ and $H_{i+1}$ is obtained from $H_{i}$ by adding a vertex $u \in V(G) \backslash V\left(H_{i}\right)$ to a vertex $v_{j} \in V\left(H_{i}\right)$ such that $\operatorname{col}\left(u v_{j}\right) \notin N_{H_{i}}^{c}\left(v_{j}\right)$.

To limit the possibly many choices for $u$ and $v_{j}$, we choose a vertex $v_{j}$ with $j$ as small as possible. Since $G$ has a finite number of vertices, Algorithm 1 will eventually stop when no vertex in $V(G) \backslash V\left(H_{S}\right)$ can increase the color degree of any vertex in $H_{s}$.

Algorithm 1 implies the following statements:

(a) $H_{0}, H_{1}, \ldots, H_{s}$ are PC unicyclic graphs (See Fig. 6).

(b) for each vertex $v \in V\left(H_{S}\right), d_{G\left[H_{s}\right]}^{c}(v)=d_{G}^{c}(v)$.

Let $H=H_{s}$. Then $V(H)=\left\{v_{1}, v_{2}, \ldots, v_{\ell+s}\right\}$ and $|V(H)|=|E(H)|$. Now for each vertex $v \in V(H)$ and each color $\alpha \in N_{G[H]}^{c}(v) \backslash N_{H}^{c}(v)$, choose the smallest number $j \in[1, s+\ell]$ such that $\operatorname{col}\left(v v_{j}\right)=\alpha$ and let $e_{v}^{\alpha}=v v_{j}$. For each vertex $v \in V(H)$, define

$$
E_{v}=\left\{e_{v}^{\alpha} \mid \alpha \in N_{G[H]}^{c}(v) \backslash N_{H}^{c}(v)\right\}
$$

Let

$$
E^{*}=\left\{u v \in E(G[H]) \mid E_{u} \cap E_{v} \neq \emptyset\right\}
$$

and

$$
E_{0}=\left\{u v \in E(G[H]) \mid u v \notin E_{u} \cup E_{v} \cup E(H)\right\} .
$$


Let $H^{\prime}$ be the spanning subgraph of $H$ with $E\left(H^{\prime}\right)=E(H) \backslash E(C)$. Then $H^{\prime}$ can be regarded as a collection of $\ell$ rooted trees such that $v_{1}, v_{2}, \ldots, v_{\ell}$ are the roots of these trees, respectively. Hence, each vertex $u$ of $H^{\prime}$ is connected by a unique path to the root of the tree that $u$ belongs to. Let $P_{u}$ denote that path for each vertex $u$ in $H^{\prime}$, and let $\operatorname{root}(u)=V\left(P_{u}\right) \cap V(C)$.

We first prove the following claim.

Claim $1 E^{*} \subseteq E_{G}(V(H) \backslash V(C), V(C))$.

Proof If $E^{*}=\emptyset$, then there is nothing to prove. Now let $u v$ be an edge in $E^{*}$. Then $u v \in E_{u} \cap E_{v} \subseteq E(G[H])$. If $u, v \in V(C)$, then $\left\{u v, u C^{+} v, u C^{-} v\right\}$ is a PC theta graph, a contradiction. If $u, v \in V(H) \backslash V(C)$, then either $u \notin V\left(P_{v}\right)$ or $v \notin V\left(P_{u}\right)$. Without loss of generality, assume that $v \notin V\left(P_{u}\right)$ and $P_{u}=u u_{1} u_{2} \ldots u_{p}$ with $u_{p} \in V(C)$ and $p \geq 1$. Apply Lemma 5.6 to $C, P_{u}$ and $v$. We obtain a PC path $Q=u w_{1} w_{2} \ldots w_{q} v$ such that $V(C) \subset V(Q), w_{q} \in V(C)$ and $w_{1} \in V(C) \cup\left\{u_{1}\right\}$. Recall that $u v \in E^{*}$. By Algorithm 1 and the choice of $e_{u}^{\operatorname{col}(u v)}$ and $e_{v}^{c o l(u v)}$, we know that $\operatorname{col}(u v) \notin \operatorname{col}(u, C) \cup \operatorname{col}(v, C) \cup\left\{\operatorname{col}\left(u u_{1}\right)\right\}$. In particular, $\operatorname{col}(u v) \neq \operatorname{col}\left(u w_{1}\right)$ and $\operatorname{col}(u v) \neq \operatorname{col}\left(w_{q} v\right)$. Thus $u Q v u$ is a PC cycle containing $C$ and longer than $C$. This contradicts that $C$ is a maximal PC cycle. Hence we get $E^{*} \subseteq E_{G}((V(H) \backslash$ $V(C), V(C))$.

Claim 2 Either $H=C$ or $\left|E_{0} \cap E_{G}(u, C)\right| \geq\left|E^{*} \cap E_{G}(u, C)\right|+1$ for each vertex $u \in V(H) \backslash V(C)$.

Proof Suppose that $H \neq C$. For each vertex $u \in V(H) \backslash V(C)$, define

$$
T_{u}=\left\{v \in V(C) \mid u v \in E^{*}\right\} .
$$

If $\operatorname{root}(u) \in T_{u}$ for some vertex $u \in V(H) \backslash V(C)$, then $\left|P_{u}\right| \geq 3$. By the construction of $H$, we can assume that $P_{u}=u u_{1} u_{2} \ldots u_{p}, u=v_{h}$ and $u_{p}=v_{j}$ for some $p \geq 2$, $h \in[\ell+1, n]$ and $j \in[1, \ell]$. Then $v_{h}$ is the unique vertex in $V\left(H_{h-\ell}\right) \backslash V\left(H_{h-\ell-1}\right)$, $\operatorname{root}(u)=v_{j}$ and $u v_{j} \in E^{*}$. Thus $u v_{j}=e_{v_{j}}^{\operatorname{col}\left(u v_{j}\right)}=e_{u}^{\operatorname{col}\left(u v_{j}\right)}$. By the choice of $e_{v_{j}}^{\operatorname{col}\left(u v_{j}\right)}$, we know that $\operatorname{col}\left(u v_{j}\right) \in N_{G\left[H_{h-\ell-1]}\right.}^{c}\left(v_{j}\right)$. This means that $S_{v_{j}}^{h-\ell-1} \neq \emptyset$. So, when we reach the state of $H_{h-\ell-1}$ in Algorithm 1, we should add a vertex to $v_{j}$ or to some $v_{i}$ with $1 \leq i<j$, but not to $u_{1}$. This contradicts with the structure of $H_{h-\ell}$. Thus $\operatorname{root}(u) \notin T_{u}$ for each vertex $u \in V(H) \backslash V(C)$.

Now let $x_{0}=\operatorname{root}(u), P_{u}=u u_{1} u_{2} \ldots u_{p}(p \geq 1)$ and $k=\left|T_{u}\right|$. Choose a direction for $C$. When $k \geq 1$, let $x_{i+1}$ be the vertex in $T_{u}$ firstly appearing after $x_{i}$ along this direction for all $i \in[0, k-1]$. Then $T_{u}=\left\{x_{i} \mid i \in[1, k]\right\}$ (See Fig. 7). By the construction of $H$ and the definition of $E^{*}$, we know that $\operatorname{col}(u v) \neq \operatorname{col}(u w)$ for each pair of distinct vertices $v, w \in T_{u} \cup\left\{u_{1}\right\}$. We will prove that there exists a vertex $y_{i} \in V\left(x_{i} C^{+} x_{i+1}\right)$ (indices are taken module $\left.k+1\right)$ such that $u y_{i} \in E_{0}$ for all $i \in[0, k]$.

Case $1 k \geq 1$. 
Fig. 7 The structure between $u$ and $C$

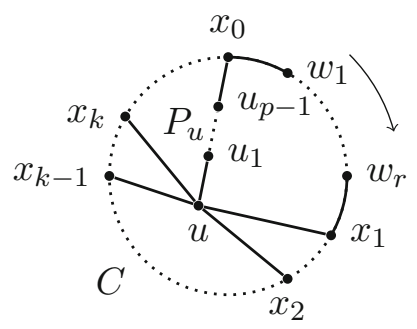

In this case, $T_{u} \neq \varnothing$. Firstly, we will prove the existence of $y_{0}$. Since the cycle $u P_{u} x_{0} C^{-} x_{0}^{+} u$ is not a PC cycle, $x_{0}^{+} \neq x_{1}$. Hence we can assume that $x_{0} C^{+} x_{1}$ $=x_{0} w_{1} w_{2} \cdots w_{r} x_{1}$ with $r \geq 1$ (See Fig. 7). Let $\alpha=\operatorname{col}\left(u u_{1}\right), x_{0}=w_{0}$ and $x_{1}=w_{r+1}$.

If $\operatorname{col}\left(u w_{i}\right)=\alpha$ for all $i \in[1, r]$, then by considering the cycle $u w_{r} C^{-} x_{1}$ and the fact that $\operatorname{col}\left(u x_{1}\right) \neq \alpha$, we get $\operatorname{col}\left(u w_{r}\right)=\operatorname{col}\left(w_{r} w_{r-1}\right)$. Thus $\alpha=\operatorname{col}\left(u u_{1}\right)$ $=\operatorname{col}\left(u w_{r}\right)=\operatorname{col}\left(w_{r} w_{r-1}\right)$. Note that $u u_{1}, w_{r} w_{r-1} \in E(H)$. By the choices of $E_{u}$ and $E_{w_{r}}$, we know that $u w_{r} \in E_{0}$. Thus we can choose $y_{0}=w_{r}$.

Otherwise, $\left\{\operatorname{col}\left(u w_{i}\right) \mid i \in[1, r]\right\} \nsubseteq\{\alpha\}$. Let $t$ be the smallest integer in $[1, r]$ such that $\operatorname{col}\left(u w_{t}\right) \neq \alpha$. Suppose that there is no choice for $y_{0}$. We assert that $\operatorname{col}\left(u w_{t}\right)$ $s=\operatorname{col}\left(w_{t} w_{t+1}\right)$. If $t=1$, then we get $\operatorname{col}\left(u w_{1}\right)=\operatorname{col}\left(w_{1} w_{2}\right)$ by considering the cycle $u P_{u} x_{0} C^{-} w_{1} u$. If $t \geq 2$, then $\operatorname{col}\left(w_{t-2} w_{t-1}\right) \neq \alpha$ (otherwise, $u w_{t-1} \in E_{0}$ ). By considering the cycle $u w_{t-1} C^{-} w_{t} u$, we get $\operatorname{col}\left(u w_{t}\right)=\operatorname{col}\left(w_{t} w_{t+1}\right)$. Note that $u w_{t} \notin E_{0}$. This implies that $u w_{t} \in E_{u}$. Since $u w_{t+1} \notin E_{0}$ and $u w_{t} C^{-} w_{t+1} u$ is not a PC cycle, we get $\operatorname{col}\left(u w_{t+1}\right)=\operatorname{col}\left(w_{t+1} w_{t+1}^{+}\right)$. Repeat this process. We finally get $\operatorname{col}\left(u x_{1}\right)=\operatorname{col}\left(x_{1} x_{1}^{+}\right)$. This contradicts that $u x_{1} \in E^{*}$. So $y_{0}$ exists when $\left\{\operatorname{col}\left(u w_{i}\right) \mid\right.$ $i \in[1, r]\} \nsubseteq\{\alpha\}$.

The existence proof for $y_{i}$ when $i \in[1, k]$ is similar, and therefore omitted.

Case $2 k=0$.

In this case, $T_{u}=\emptyset$. Let $\alpha=\operatorname{col}\left(u u_{1}\right)$ and $x_{0} C^{+} x_{0}=x_{0} w_{1} w_{2} \cdots w_{\ell-1} x_{0}$. Since $d^{c}(u) \geq \frac{n+1}{2}$ and $\ell \geq \frac{n+1}{2}$, we get $\left\{\operatorname{col}\left(u w_{i}\right) \mid i \in[1, \ell-1]\right\} \nsubseteq\{\alpha\}$. Let $t$ be a smallest integer in $[1, \ell-1]$ such that $\operatorname{col}\left(u w_{t}\right) \neq \alpha$. Similar to the proof in the case that $k \geq 1$, there must exist a vertex $y_{0} \in\left\{w_{1}, w_{2}, \cdots, w_{\ell-1}\right\}$ such that $u y_{0} \in E_{0}$.

In summary, $\left|E_{0} \cap E_{G}(u, C)\right| \geq\left|E^{*} \cap E_{G}(u, C)\right|+1$ for each vertex $u \in V(H) \backslash$ $V(C)$. This completes the proof of Claim 2.

We continue the proof of Theorem 5.4 by counting the number of edges of $G[H]$. We have

$$
\sum_{v \in V(H)}\left|E_{v}\right|+|E(H)|+\left|E_{0}\right|-\left|E^{*}\right|=\left(\begin{array}{c}
|V(H)| \\
2
\end{array}\right) .
$$

Note that for each vertex $v \in V(H)$,

$$
\left|E_{v}\right|+d_{H}(v)=d_{G[H]}^{c}(v)=d_{G}^{c}(v) .
$$


Summing over all $v \in V(H)$, we obtain

$$
\sum_{v \in V(H)}\left|E_{v}\right|+2|E(H)|=\sum_{v \in V(H)} d_{G}^{c}(v)
$$

Recall that $|V(H)|=|E(H)|$. By using this and combining (2) and (3), we obtain

$$
\left|E^{*}\right|=\left|E_{0}\right|+\sum_{v \in V(H)} d_{G}^{c}(v)-\frac{|V(H)|(|V(H)|+1)}{2} \geq\left|E_{0}\right| .
$$

If $H \neq C$, then $|V(H) \backslash V(C)| \geq 1$. By Claims 1 and 2 , we get

$$
\begin{aligned}
\left|E^{*}\right| & =\sum_{v \in V(H) \backslash V(C)}\left|E^{*} \cap E_{G}(v, C)\right| \leq \sum_{v \in V(H) \backslash V(C)}\left(\left|E_{0} \cap E_{G}(v, C)\right|-1\right) \\
& \leq\left|E_{0}\right|-1 .
\end{aligned}
$$

This contradicts (4). So $H=C$. By applying Claim 1 , we get $E^{*}=\varnothing$. According to (4), we further obtain $E_{0}=\emptyset$ and

$$
\sum_{v \in V(H)} d_{G}^{c}(v)=\frac{|V(H)|(|V(H)|+1)}{2} .
$$

Note that $\delta^{c}(G) \geq \frac{n+1}{2}$ and $|V(H)|=\ell \leq n$. This implies that $C$ is a PC Hamilton cycle and $d_{G}^{c}(v)=\frac{n+1}{2}$ for each vertex $v \in V(G)$. This completes the proof of Theorem 5.4.

\section{Concluding Remarks and Questions}

Cycles in multipartite tournaments have attracted much attention during the past decades. See, e.g., the survey paper due to Volkmann [22].

We conclude this paper with a slight strengthening of our main theorem, by involving a connectivity condition for the essentially multipartite tournaments in Theorem 1.4. We first need an additional definition. Let $G$ be a colored complete graph. Let $\mathcal{F}_{G}$ be the set of mappings such that for each $f \in \mathcal{F}_{G}$ and each edge $u v \in E(G)$, we have $\operatorname{col}(u v)=f(u)$ or $\operatorname{col}(u v)=f(v)$. Clearly, $\mathcal{F}_{G} \neq \emptyset$ if and only if $G$ is essentially a multipartite tournament. For each $f \in \mathcal{F}_{G}$, we can construct a unique multipartite tournament $D_{G}^{f}$ using Construction 1.3. Now we say that a colored complete graph $G$ is essentially a strongly connected multipartite tournament if $\mathcal{F}_{G} \neq \emptyset$ and for each mapping $f \in \mathcal{F}_{G}$, the multipartite tournament $D_{G}^{f}$ is strongly connected. Considering this definition, we can slightly improve Theorem 1.4, as follows.

Theorem 6.1 Let $G$ be a CD-critical colored complete graph. Then G contains no PC theta graph if and only if $G$ is essentially a strongly connected multipartite tournament, unless $G$ is a colored $K_{2}$ or a colored $K_{4}$ containing a monochromatic edge-cut. 
Proof The sufficiency follows obviously from Observation 1.1.

For the necessity, we first deal with the case that $\delta^{c}(G) \leq 2$. If $G$ is neither a colored $K_{2}$ nor a colored $K_{4}$ containing a monochromatic edge-cut, then by Observation 1.3, $G$ must be isomorphic to the graph in Fig. 1c. In that case, the only PC cycle of length 4 in $G$ is a directed Hamilton cycle in $D_{G}^{f}$ for each $f \in \mathcal{F}_{G}$. Hence, $D_{G}^{f}$ is always strongly connected, i.e., $G$ is essentially a strongly connected multipartite tournament. When $\delta^{c}(G) \geq 3$, by Theorem 1.4 , we know that $\mathcal{F}_{G} \neq \varnothing$. Suppose, to the contrary, that $G$ is not essentially a strongly connected multipartite tournament. Then there exists a mapping $f \in \mathcal{F}_{G}$ such that $D_{G}^{f}$ is not strongly connected. Let $\left(S_{1}, S_{2}\right)$ be a partition of $V\left(D_{G}^{f}\right)$ such that $(i) S_{1}, S_{2} \neq \emptyset$; ( $\left.i i\right)$ all the arcs between $S_{1}$ and $S_{2}$ have heads in $S_{1}$; (iii) $\left|S_{2}\right|$ is as small as possible. Since $G$ is CD-critical and $S_{1} \neq \emptyset$, we have $\delta^{c}\left(G\left[S_{2}\right]\right)<\delta^{c}(G)$. Note that $\operatorname{col}\left(S_{1}, v\right) \subseteq\{f(v)\}$ for each vertex $v \in S_{2}$. There must exist a vertex $x \in S_{2}$ such that $f(x) \notin N_{G\left[S_{2}\right]}^{c}(x)$. Thus for each vertex $u \in S_{2} \backslash\{x\}$, we have $f(u) \neq f(x)$ and $\operatorname{col}(x u)=f(u)$, i.e., $u x \in A\left(D_{G}^{f}\right)$. Since $\delta^{c}(G) \geq 3$, we have $\left|S_{2} \backslash\{x\}\right| \geq 2$. Now $\left(S_{1} \cup\{x\}, S_{2} \backslash\{x\}\right)$ is a partition of $D_{G}^{f}$ satisfying $(i)$, (ii) and $\left|S_{2} \backslash\{x\}\right|<\left|S_{2}\right|$, a contradiction.

This completes the proof of Theorem 6.1.

By the proofs of Observations 1.2 and 3.3 in [14] and [6], respectively, we know that if $C$ is a PC cycle in a colored complete graph (complete bipartite graph), then each vertex of $C$ is contained in a PC cycle of length 3 or 4 (4 or 6). In fact, by a similar argument, we can prove that if $C$ is a $\mathrm{PC}$ cycle in a colored complete multipartite graph, then each vertex of $C$ is contained in a PC cycle of length $\ell \in\{3,4,6\}$ (see Observation 1.9 in [13]). Are there similar results for PC theta graphs? In particular, what is the answer to the following question?

Question 6.1 Does there exist a constant $t$ such that each colored complete graph $G$ which contains a PC theta graph contains a PC theta graph of order at most $t$ ? What is the answer to this question if we impose that $\delta^{c}(G) \geq 3$ ?

Theorem 5.4 implies a possible approach for proving the existence of a PC Hamilton cycle under the condition of Conjecture 5.1. This also leads to the following question.

Question 6.2 Let $\Theta_{k, \ell, m}$ be a PC theta graph in a colored complete graph $G$. Which conditions on $G$ imply that there exists a $\mathrm{PC}$ cycle $C$ in $G$ with $|C|>\max$ $\{k+\ell, \ell+m, m+k\}$ ?

Open Access This article is distributed under the terms of the Creative Commons Attribution 4.0 International License (http://creativecommons.org/licenses/by/4.0/), which permits unrestricted use, distribution, and reproduction in any medium, provided you give appropriate credit to the original author(s) and the source, provide a link to the Creative Commons license, and indicate if changes were made.

\section{References}

1. Alon, N., Gutin, G.: Properly colored Hamilton cycles in edge-colored complete graphs. Random Struct. Algorithms 11, 179-186 (1997) 
2. Bollobás, B., Erdős, P.: Alternating Hamiltonian cycles. Israel J. Math. 23, 126-131 (1976)

3. Bondy, J.A., Murty, U.S.R.: Graph Theory, Springer Graduate Texts in Mathematics, vol. 244 (2008)

4. Chen, C.C., Daykin, D.E.: Graphs with Hamiltonian cycles having adjacent lines different colors. J. Comb. Theory Ser. B 21, 135-139 (1976)

5. Daykin, D.E.: Graphs with cycles having adjacent lines of different colors. J. Comb. Theory Ser. B 20, 149-152 (1976)

6. Fujita, S., Li, R., Zhang, S.: Color degree and monochromatic degree conditions for short properly colored cycles. J. Graph Theory 87, 362-373 (2018)

7. Fujita, S., Magnant, C.: Properly colored paths and cycles. Discrete Appl. Math. 159, 1391-1397 (2011)

8. Gallai, T.: Transitiv orientierbare graphen. Acta Math. Hung. 18, 25-66 (1967)

9. Grossman, J.W., Häggkvist, R.: Alternating cycles in edge-partitioned graphs. J. Comb. Theory Ser. B 34, 77-81 (1983)

10. Gyárfás, A., Simony, G.: Edge colorings of complete graphs without tricolored triangles. J. Graph Theory 46, 211-216 (2004)

11. Li, B., Ning, B., Xu, C., Zhang, S.: Rainbow triangles in edge-colored graphs. Eur. J. Comb. 36, 453-459 (2014)

12. Li, H.: Rainbow $C_{3}$ 's and $C_{4}$ 's in edge-colored graphs. Discrete Math. 313, 1893-1896 (2013)

13. Li, R.: Properly colored cycles in edge-colored graphs (PhD-Thesis), Enschede, 153 pp. (2018); https:// doi.org/10.3990/1.9789036544719

14. Li, R., Broersma, H.J., Zhang, S.: Vertex disjoint properly edge-colored cycles in edge-colored complete graphs, arXiv:1708.08641 [math.CO]

15. Li, R., Broersma, H.J., Xu, C., Zhang, S.: Cycle extension in edge-colored complete graphs. Discrete Math. 340, 1235-1241 (2017)

16. Li, R., Ning, B., Zhang, S.: Color degree sum conditions for rainbow triangles in edge-colored graphs. Graphs Comb. 32, 2001-2008 (2016)

17. Lo, A.: A Dirac type condition for properly coloured paths and cycles. J. Graph Theory 76, 60-87 (2014)

18. Lo, A.: An edge-coloured version of Dirac's theorem. SIAM J. Discrete Math. 28, 18-36 (2014)

19. Lo, A.: Properly coloured Hamiltonian cycles in edge-coloured complete graphs. Combinatorica 36, 471-492 (2016)

20. Manoussakis, Y., Spyratos, M., Tuza, Zs, Voigt, M.: Minimal colorings for properly colored subgraphs. Graphs Comb. 12, 345-360 (1996)

21. Shearer, J.: A property of the colored complete graph. Discrete Math. 25, 175-178 (1979)

22. Volkmann, L.: Multipartite tournaments: a survey. Discrete Math. 307, 3097-3129 (2007)

23. Yeo, A.: A note on alternating cycles in edge-colored graphs. J. Comb. Theory Ser. B 69, 222-225 (1997) 